\title{
Merging Photoredox with Palladium Catalysis: Decarboxylative ortho-Acylation of Acetanilides with $\alpha$-Oxocarboxylic Acids under Mild Reaction Conditions
}

\author{
Chao Zhou, Pinhua Li*,†, Xianjin Zhu, and Lei Wang*,†,\$ \\ ${ }^{\dagger}$ Department of Chemistry, Huaibei Normal University, Huaibei, Anhui 235000, P R \\ China, Tel:+86-561-3802-069; Fax: +86-561-3090-518 \\ E-mail: pphuali@126.com; leiwang88@hotmail.com \\ \$State Key Laboratory of Organometallic Chemistry, Shanghai Institute of Organic \\ Chemistry, Chinese Academy of Sciences, Shanghai 200032, P R China
}

\section{Table of Contents for Supporting Information}

1. General considerations.................................................

2. Representative procedure for the reaction...............................2

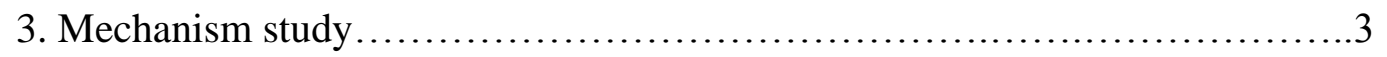

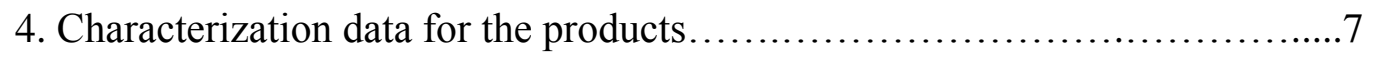

5. ${ }^{1} \mathrm{H}$ and ${ }^{13} \mathrm{C}$ NMR spectra of the products............................... 19

6. Reference........................................................ 51 


\section{General considerations}

The ${ }^{1} \mathrm{H}$ NMR and ${ }^{13} \mathrm{C}$ NMR spectra were recorded on a $400 \mathrm{MHz}$ Bruker FT-NMR spectrometers (400 MHz or $100 \mathrm{MHz}$, respectively). All chemical shifts are given as $\delta$ value (ppm) with reference to tetramethylsilane (TMS) as an internal standard. The peak patterns are indicated as follows: s, singlet; $d$, doublet; t, triplet; $\mathrm{m}$, multiplet; q, quartet. The coupling constants, $J$, are reported in Hertz (Hz). High resolution mass spectroscopy data of the product were collected on an Agilent Technologies 6540 UHD Accurate-Mass Q-TOF LC/MS (ESI).

Acetanilides were prepared according to the reported methods, ${ }^{[1]}$ and $\alpha$-oxocarboxylic acids were prepared according to the literature. ${ }^{[2]}$ The chemicals and solvents were purchased from commercial suppliers Aldrich, USA, or Shanghai Chemical Company, China. Products were purified by flash chromatography on 200-300 mesh silica gels, $\mathrm{SiO}_{2}$.

\section{Representative procedure for the reaction}<smiles>CC(=O)Nc1ccccc1</smiles>

$1 \mathrm{a}$<smiles>O=C(O)C(=O)c1ccccc1</smiles>

$2 a$
$\mathrm{Pd}(\mathrm{OAc})_{2}(5 \mathrm{~mol} \%)$

$\underset{\mathrm{O}_{2} \text { balloon, } \mathrm{PhCl}, \mathrm{rt}}{\stackrel{\text { Eosin } \mathrm{Y}(3 \mathrm{~mol} \%)}{\longrightarrow}}$ $3 \mathrm{~W}$ Green LED, $15 \mathrm{~h}$<smiles>CC(=O)Nc1ccccc1C(=O)c1ccccc1</smiles>

$3 a$

Acetanilide (1a, $0.50 \mathrm{mmol})$, phenylglyoxylic acid (2a, $0.75 \mathrm{mmol}), \mathrm{Pd}(\mathrm{OAc})_{2}$ (5.0 mol \%), Eosin $\mathrm{Y}(3.0 \mathrm{~mol} \%)$ and chlorobenzene $(2.0 \mathrm{~mL})$ was added to an oven-dried reaction vessel equipped with magnetic stirring bar, and the reaction vessel was irradiated using $3 \mathrm{~W}$ green LED under $\mathrm{O}_{2}$ atmosphere for $15 \mathrm{~h}$. After the reaction was completed, the reaction solution was concentrated under reduced pressure to yield crude product, which was purified by flash chromatography (silica gel, petroleum ether/ethyl acetate $=9: 1$ ) to give the desired product 3a in $80 \%$ yield as a white solid. 


\section{Mechanism study}

\subsection{Determination of superoxide radical anion}

Superoxide radical anion $\left(\mathrm{O}_{2}^{-} \cdot\right)$ is generated form molecular oxygen by single electron transfer (SET). ${ }^{[3]}$ 5,5-Dimethyl-1-pyrroline- $N$-oxide (DMPO) was used as a probe to capture active species $\mathrm{O}_{2}^{-} \cdot{ }^{[4]}$ As shown in Figure 1, when the solution of DMPO, 2a and Eosin Y in chlorobenzene solution without irradiation of green LED, no signal was detected. In contrast, when the same solution was irradiated with green LED, a single radical was trapped, the characteristic signal of $\mathrm{O}_{2}^{-} \cdot$ was clearly observed.

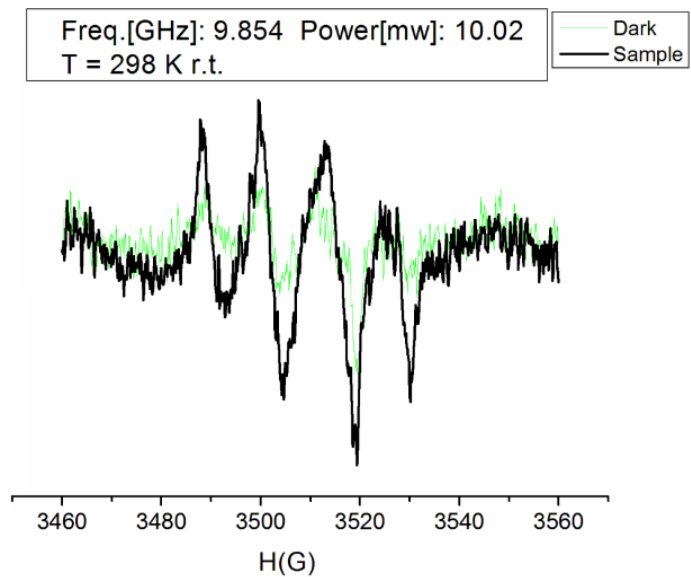

Figure 1. ESR spectrum of air-saturated chlorobenzene solution of $2 \mathrm{a}\left(1.0 \times 10^{-3} \mathrm{M}\right)$, Eosin $\mathrm{Y}(3$ mol \%), and DMPO $\left(2.0 \times 10^{-2} \mathrm{M}\right)$ without irradiation of green LED; $2 \mathrm{a}\left(1.0 \times 10^{-3} \mathrm{M}\right)$, Eosin $\mathrm{Y}(3$ mol \%), and DMPO $\left(2.0 \times 10^{-2} \mathrm{M}\right)$ upon irradiation by green LED for $30 \mathrm{~s}$.

\subsection{Free radical-trapping experiment}

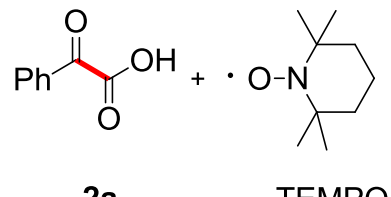

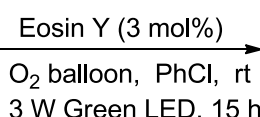

$3 \mathrm{~W}$ Green LED, $15 \mathrm{~h}$

$2 a$

TEMPO

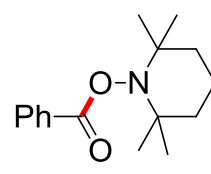

5

Phenylglyoxylic acid (2a, $45.0 \mathrm{mg}, \quad 0.3 \mathrm{mmol})$, 2,2,6,6-Tetramethyl-1Oxylpiperidine (TEMPO, $78.1 \mathrm{mg}, 0.5 \mathrm{mmol})$ and Eosin $\mathrm{Y}(5.8 \mathrm{mg}, 0.009 \mathrm{mmol})$ were dissolved in chlorobenzene $(2.0 \mathrm{~mL})$ in an oven-dried reaction vessel equipped 
with magnetic stirring bar, and the reaction vessel was irradiated using $3 \mathrm{~W}$ green LED under the $\mathrm{O}_{2}$ atmosphere for $15 \mathrm{~h}$. After the reaction was carried out, concentrated under reduced pressure to yield the crude product, which was purified by flash chromatography (silica gel, petroleum ether/ethyl acetate $=20: 1$ to 10:1), affording the desired product $\mathbf{5}$ as a colorless solid (53.3 $\mathrm{mg}, 68 \%$ yield).

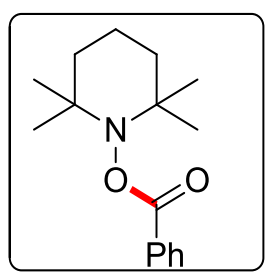

2,2,6,6-Tetramethylpiperidin-1-yl benzoate (5): ${ }^{[5]}$ White solid (53.3 mg, 68\% yield); ${ }^{1} \mathrm{H}$ NMR $\left(400 \mathrm{MHz}, \mathrm{CDCl}_{3}\right) \delta: 8.08(\mathrm{~d}, J=7.6 \mathrm{~Hz}, 2 \mathrm{H}), 7.56-7.54(\mathrm{~m}, 1 \mathrm{H})$, 7.47-7.43 (m, 2H), 1.81-1.75 (m, 2H), 1.70-1.67 (m, 1H), 1.59-1.56 (m, 2H), 1.47-1.44 (m, 1H), $1.27(\mathrm{~s}, 6 \mathrm{H}), 1.13(\mathrm{~s}, 6 \mathrm{H}) ;{ }^{13} \mathrm{C} \mathrm{NMR}\left(100 \mathrm{MHz}, \mathrm{CDCl}_{3}\right) \delta: 166.03$, 132.56, 129.48, 129.24, 128.17, 60.09, 38.82, 31.69, 20.58, 16.74. HRMS (ESI) $\left([\mathrm{M}+\mathrm{H}]^{+}\right)$Calcd. For $\mathrm{C}_{16} \mathrm{H}_{24} \mathrm{NO}_{2}: 262.1807$, Found: 262.1803 .

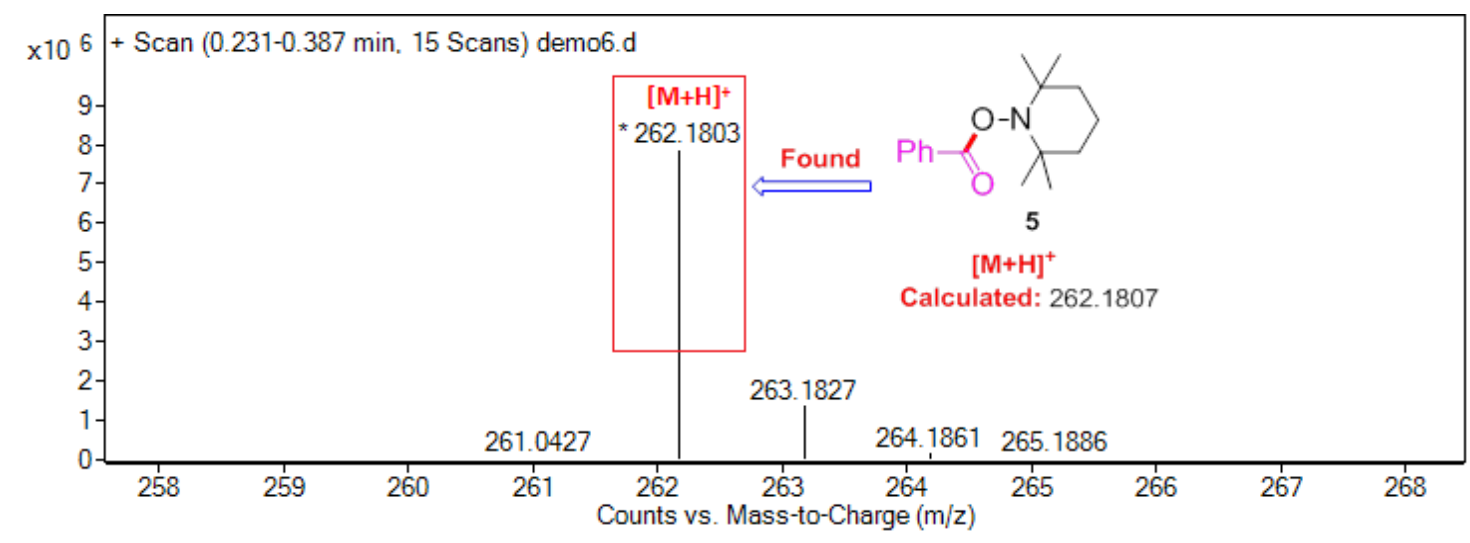




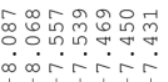

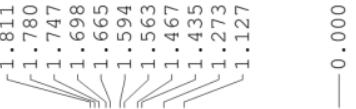
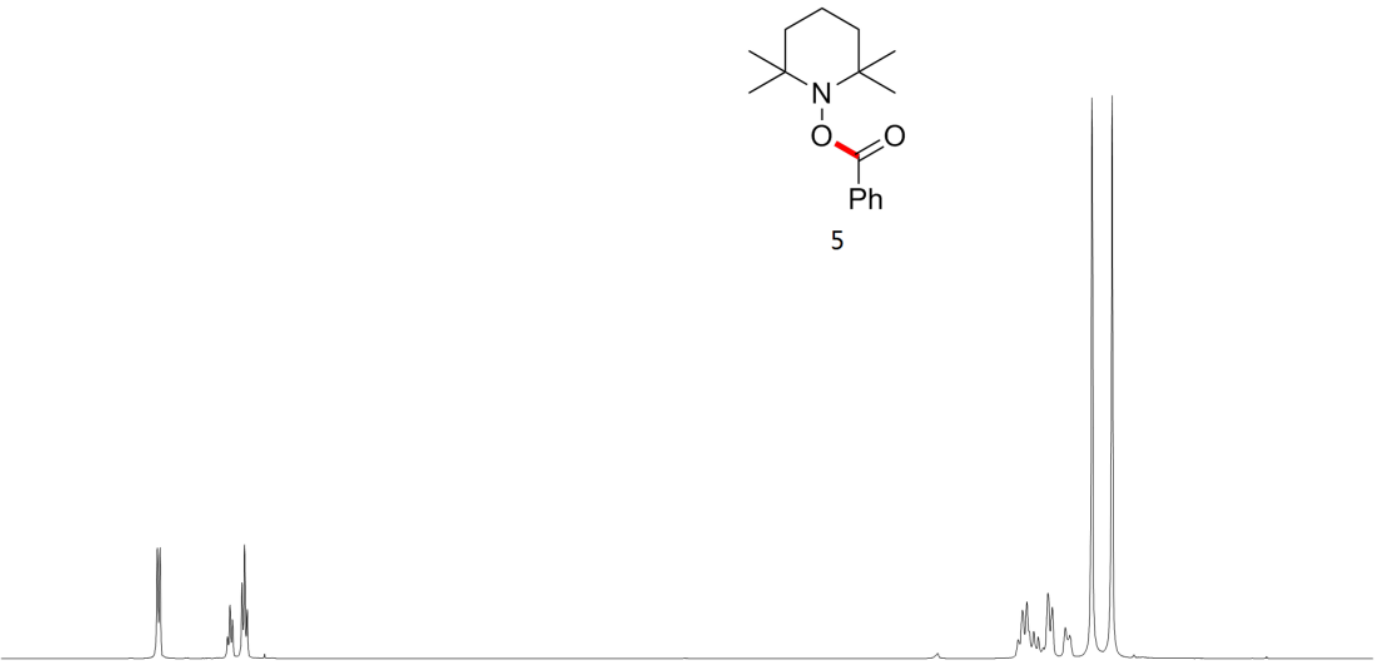

$\begin{array}{llllllllllllllllllll}9.0 & 8.5 & 8.0 & 7.5 & 7.0 & 6.5 & 6.0 & 5.5 & 5.0 & 4.5 & 4.0 & 3.5 & 3.0 & 2.5 & 2.0 & 1.5 & 1.0 & 0.5 & 0.0 & \mathrm{ppm}\end{array}$

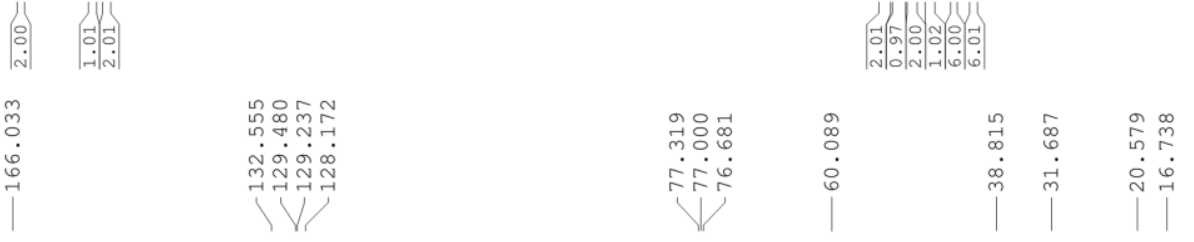

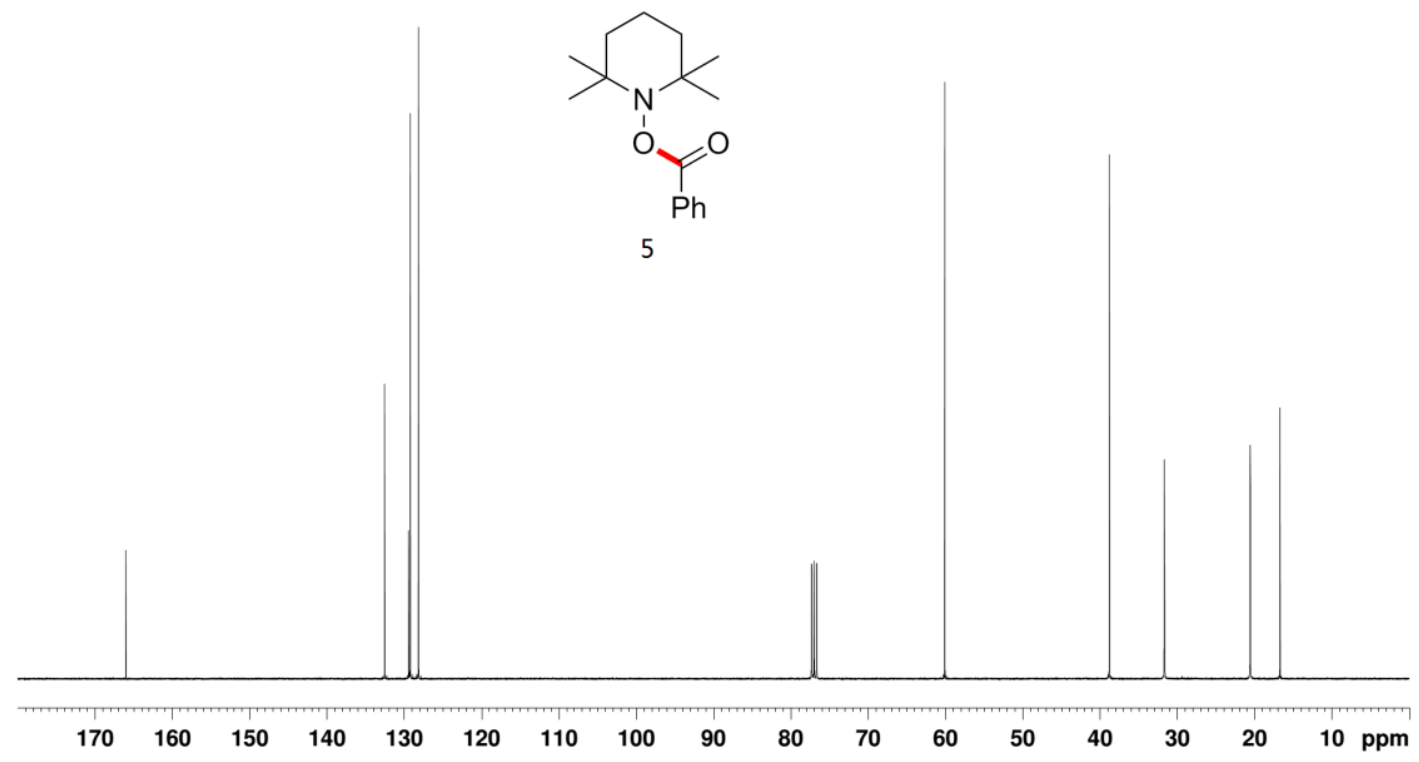




\subsection{Typical procedure for $\mathrm{CO}_{2}$ detection by FT-IR}

Acetanilide (1a, $0.5 \mathrm{mmol})$, phenylglyoxylic acid (2a, $0.75 \mathrm{mmol}), \mathrm{Pd}(\mathrm{OAc})_{2}(5$ mol \%), Eosin Y (3 mol \%) and chlorobenzene (2 $\mathrm{mL})$ was added to an Schlenk tube equipped with magnetic stirring bar, and the reaction vessel was irradiated using $3 \mathrm{~W}$ green LED under the $\mathrm{O}_{2}$ atmosphere for $15 \mathrm{~h}$. After completion of the reaction, the resulting gas from the reaction mixture was directly determined by FT-IR analysis (Figure 2), and the concentration of $\mathrm{CO}_{2}$ was found to be $3935.82 \mathrm{ppm}$.

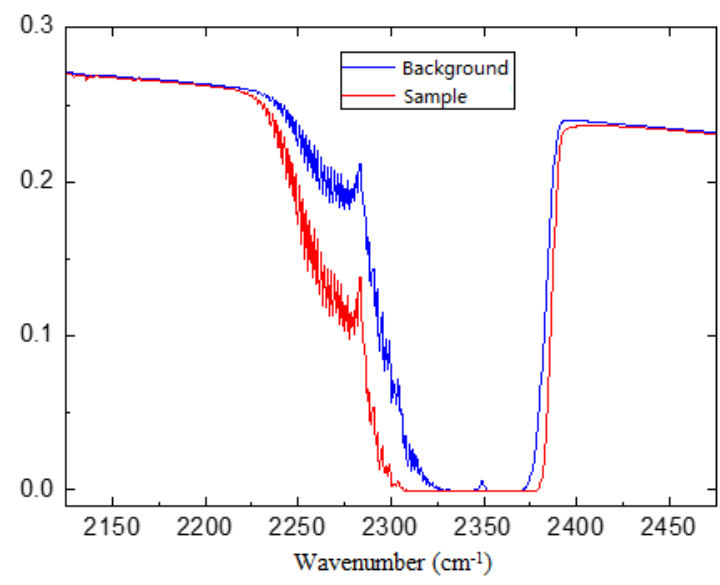

Figure 2. FT-IR analysis of the resulting gas by a Bruker Tensor 27 FT-IR 


\section{Characterization data for the products}

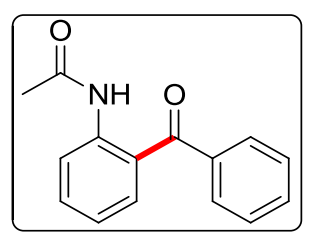

$N$-(2-Benzoylphenyl)acetamide (3a): ${ }^{[6]}$ White solid; $95.6 \mathrm{mg}, 80 \%$ yield; ${ }^{1} \mathrm{H}$ NMR $\left(400 \mathrm{MHz}, \mathrm{CDCl}_{3}\right) \delta: 10.81(\mathrm{~s}, 1 \mathrm{H}), 8.63(\mathrm{~d}, J=8.4 \mathrm{~Hz}, 1 \mathrm{H}), 7.70(\mathrm{~d}, J=7.6 \mathrm{~Hz}, 2 \mathrm{H})$, 7.62-7.54 (m, 3H), 7.51-7.47 (m, 2H), $7.08(\mathrm{t}, J=7.6 \mathrm{~Hz}, 1 \mathrm{H}), 2.23(\mathrm{~s}, 3 \mathrm{H}) ;{ }^{13} \mathrm{C}$

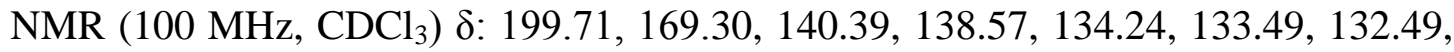
$129.86,128.30,123.25,122.07,121.54,25.22$.

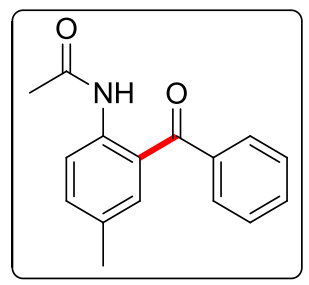

$N$-(2-Benzoyl-4-methylphenyl)acetamide $(3 \mathrm{~b}):^{[6]}$ White solid; $97.5 \mathrm{mg}, 77 \%$ yield; ${ }^{1} \mathrm{H}$ NMR $\left(400 \mathrm{MHz}, \mathrm{CDCl}_{3}\right) \delta: 10.63(\mathrm{~s}, 1 \mathrm{H}), 8.48(\mathrm{~d}, J=8.8 \mathrm{~Hz}, 1 \mathrm{H}), 7.70(\mathrm{~d}, J=$ $7.6 \mathrm{~Hz}, 2 \mathrm{H}), 7.62-7.58(\mathrm{~m}, 1 \mathrm{H}), 7.51-7.47$ (m, 2H), 7.38-7.36 (m, 1H), $7.33(\mathrm{~s}, 1 \mathrm{H})$, $2.29(\mathrm{~s}, 3 \mathrm{H}), 2.20(\mathrm{~s}, 3 \mathrm{H}) ;{ }^{13} \mathrm{C}$ NMR $\left(100 \mathrm{MHz}, \mathrm{CDCl}_{3}\right) \delta: 199.60,168.91,138.65$, 137.91, 134.79, 133.42, 132.37, 131.59, 129.80, 128.89, 128.24, 121.55, 25.09, 20.65.

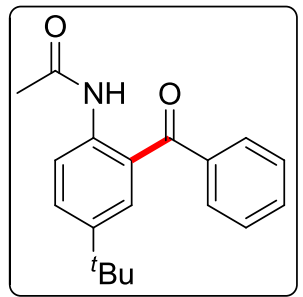

$N$-(2-Benzoyl-4-(tert-butyl)phenyl)acetamide (3c): White solid; $112.2 \mathrm{mg}, 76 \%$ yield; ${ }^{1} \mathrm{H}$ NMR $\left(400 \mathrm{MHz}, \mathrm{CDCl}_{3}\right) \delta: 10.63(\mathrm{~s}, 1 \mathrm{H}), 8.51(\mathrm{~d}, J=8.8 \mathrm{~Hz}, 1 \mathrm{H}), 7.72(\mathrm{~d}$, $J=7.6 \mathrm{~Hz}, 2 \mathrm{H}), 7.62-7.59(\mathrm{~m}, 2 \mathrm{H}), 7.56(\mathrm{~s}, 1 \mathrm{H}), 7.51-7.48(\mathrm{~m}, 2 \mathrm{H}), 2.20(\mathrm{~s}, 3 \mathrm{H})$, 1.25 (s, 9H); ${ }^{13} \mathrm{C}$ NMR (100 MHz, $\left.\mathrm{CDCl}_{3}\right) \delta: 199.59,168.93,144.85,138.61,137.70$, 132.44, 131.11, 130.12, 129.91, 128.18, 123.16, 121.36, 34.19, 30.99, 25.03. HRMS 
(ESI) $\left([\mathrm{M}+\mathrm{H}]^{+}\right)$Calcd. For $\mathrm{C}_{19} \mathrm{H}_{22} \mathrm{NO}_{2}$ : 296.1651, Found: 296.1656.

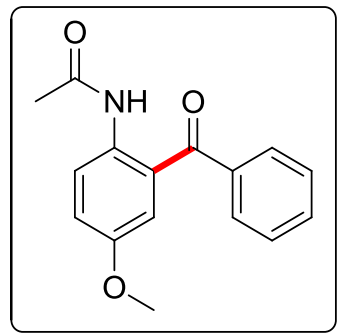

$N$-(2-Benzoyl-4-methoxyphenyl)acetamide (3d): ${ }^{[7]}$ White solid; $109.0 \mathrm{mg}, 81 \%$ yield; ${ }^{1} \mathrm{H}$ NMR (400 MHz, $\left.\mathrm{CDCl}_{3}\right) \delta: 10.30(\mathrm{~s}, 1 \mathrm{H}), 8.47$ (d, J=9.2 Hz, 1H), $7.74(\mathrm{~d}$, $J=7.4 \mathrm{~Hz}, 2 \mathrm{H}), 7.62-7.58(\mathrm{~m}, 1 \mathrm{H}), 7.51-7.47(\mathrm{~m}, 2 \mathrm{H}), 7.14-7.11(\mathrm{~m}, 1 \mathrm{H}), 7.04-7.03$ $(\mathrm{m}, 1 \mathrm{H}), 3.74(\mathrm{~s}, 3 \mathrm{H}), 2.18(\mathrm{~s}, 3 \mathrm{H}) ;{ }^{13} \mathrm{C} \mathrm{NMR}\left(100 \mathrm{MHz}, \mathrm{CDCl}_{3}\right) \delta:$ 198.94, 168.75, $154.15,138.24,133.38,132.64,129.90,128.31,125.09,123.43,119.21,118.10$, $55.58,24.91$.

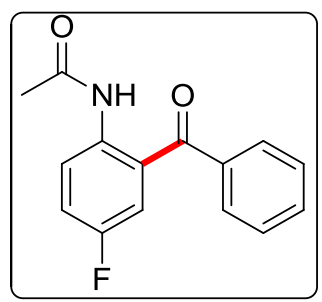

$N$-(2-Benzoyl-4-fluorophenyl)acetamide $(\mathbf{3 e}){ }^{[6]}$ White solid; $101.6 \mathrm{mg}$, $79 \%$ yield; ${ }^{1} \mathrm{H}$ NMR $\left(400 \mathrm{MHz}, \mathrm{CDCl}_{3}\right) \delta: 10.50(\mathrm{~s}, 1 \mathrm{H}), 8.61-8.58(\mathrm{~m}, 1 \mathrm{H}), 7.72(\mathrm{~d}, J=7.2 \mathrm{~Hz}$, $2 \mathrm{H}), 7.65-7.61(\mathrm{~m}, 1 \mathrm{H}), 7.53-7.50(\mathrm{~m}, 2 \mathrm{H}), 7.26-7.22(\mathrm{~m}, 2 \mathrm{H}), 2.21(\mathrm{~s}, 3 \mathrm{H}) ;{ }^{13} \mathrm{C}$ NMR $\left(100 \mathrm{MHz}, \mathrm{CDCl}_{3}\right) \delta: 198.32,168.99,156.93(\mathrm{~d}, J=242.9 \mathrm{~Hz}), 136.51(\mathrm{~d}, J=$ $2.7 \mathrm{~Hz}), 136.50,132.97,129.87,128.53,124.77(\mathrm{~d}, J=5.3 \mathrm{~Hz}), 123.71(\mathrm{~d}, J=7.1$ Hz), 120.98 (d, $J=21.8 \mathrm{~Hz}), 119.15(\mathrm{~d}, J=23.6 \mathrm{~Hz}), 25.07$.

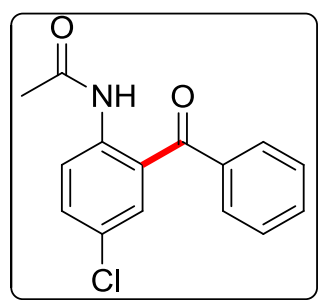

$N$-(2-Benzoyl-4-chlorophenyl)acetamide $(\mathbf{3 f}):{ }^{[7]}$ White solid; $102.4 \mathrm{mg}, 75 \%$ yield; 
${ }^{1} \mathrm{H}$ NMR (400 MHz, $\left.\mathrm{CDCl}_{3}\right) \delta: 10.62(\mathrm{~s}, 1 \mathrm{H}), 8.61(\mathrm{~d}, J=8.4 \mathrm{~Hz}, 1 \mathrm{H}), 7.72-7.70$ (m, 2H), 7.66-7.62 (m, 1H), 7.54-7.51 (m, 4H), $2.22(\mathrm{~s}, 3 \mathrm{H}) ;{ }^{13} \mathrm{C}$ NMR (100 MHz, $\left.\mathrm{CDCl}_{3}\right) \delta: 198.38,169.03,138.93,137.90,133.91,132.95,132.54,129.85,128.55$, 127.22, 124.57, 123.07, 25.16.

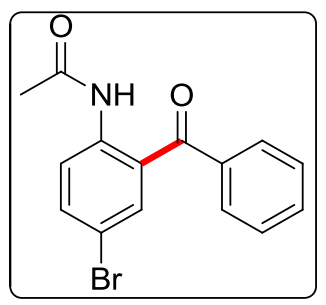

$N$-(2-Benzoyl-4-bromophenyl)acetamide (3g): ${ }^{[7]}$ White solid; $128.4 \mathrm{mg}, 81 \%$ yield; ${ }^{1} \mathrm{H}$ NMR (400 MHz, $\left.\mathrm{CDCl}_{3}\right) \delta: 10.61(\mathrm{~s}, 1 \mathrm{H}), 8.55(\mathrm{~d}, J=9.6 \mathrm{~Hz}, 1 \mathrm{H}), 7.70(\mathrm{~d}, J=$ $7.2 \mathrm{~Hz}, 2 \mathrm{H}), 7.67-7.62(\mathrm{~m}, 3 \mathrm{H}), 7.54-7.50(\mathrm{~m}, 2 \mathrm{H}), 2.22(\mathrm{~s}, 3 \mathrm{H}) ;{ }^{13} \mathrm{C}$ NMR (100 $\left.\mathrm{MHz}, \mathrm{CDCl}_{3}\right) \delta: 198.30,169.06,139.38,137.87,136.82,135.44,132.98,129.87$, $128.56,124.90,123.31,114.52,25.20$.

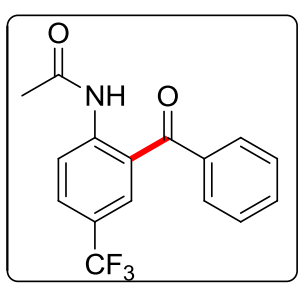

$N$-(2-Benzoyl-4-(trifluoromethyl)phenyl)acetamide (3h): White solid; $104.4 \mathrm{mg}$, $68 \%$ yield; ${ }^{1} \mathrm{H}$ NMR (400 MHz, $\left.\mathrm{CDCl}_{3}\right) \delta: 10.90(\mathrm{~s}, 1 \mathrm{H}), 8.83(\mathrm{~d}, J=8.4 \mathrm{~Hz}, 1 \mathrm{H})$, 7.82-7.80 (m, 2H), 7.71-7.69 (m, 2H), 7.66-7.64 (m, 1H), 7.55-7.52 (m, 2H), $2.26(\mathrm{~s}$, $3 \mathrm{H}) ;{ }^{13} \mathrm{C}$ NMR $\left(100 \mathrm{MHz}, \mathrm{CDCl}_{3}\right) \delta: 198.66,169.38,143.30(\mathrm{~d}, J=1.1 \mathrm{~Hz}), 137.78$, $133.16,130.75\left(\mathrm{q}, J_{1}=3.6 \mathrm{~Hz}, J_{2}=7.1 \mathrm{~Hz}\right), 130.24\left(\mathrm{q}, J_{1}=3.8 \mathrm{~Hz}, J_{2}=7.7 \mathrm{~Hz}\right)$, 129.86, 128.65, 124.89, 123.98 (d, $J=33.0 \mathrm{~Hz}), 122.67,121.61,25.30$. HRMS (ESI) $\left([\mathrm{M}+\mathrm{H}]^{+}\right)$Calcd. For $\mathrm{C}_{16} \mathrm{H}_{13} \mathrm{~F}_{3} \mathrm{NO}_{2}: 308.0898$, Found: 308.0894 . 


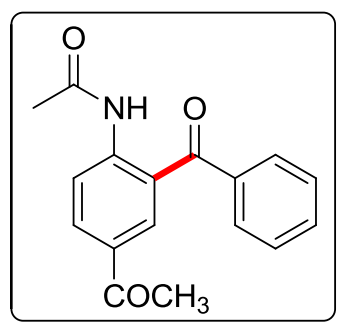

$N$-(4-Acetyl-2-benzoylphenyl)acetamide $(3 \mathbf{3 i}):{ }^{[6]}$ White solid; $115.3 \mathrm{mg}, 82 \%$ yield; ${ }^{1} \mathrm{H}$ NMR (400 MHz, $\left.\mathrm{CDCl}_{3}\right) \delta: 11.02(\mathrm{~s}, 1 \mathrm{H}), 8.79(\mathrm{~d}, J=8.8 \mathrm{~Hz}, 1 \mathrm{H}), 8.21(\mathrm{~s}, 1 \mathrm{H})$, $8.14(\mathrm{~d}, J=8.8 \mathrm{~Hz}, 1 \mathrm{H}), 7.71(\mathrm{~d}, J=7.6 \mathrm{~Hz}, 2 \mathrm{H}), 7.67-7.63(\mathrm{~m}, 1 \mathrm{H}), 7.54-7.51(\mathrm{~m}$, 2H), $2.54(\mathrm{~s}, 3 \mathrm{H}), 2.27$ (s, 3H); ${ }^{13} \mathrm{C} \mathrm{NMR}\left(100 \mathrm{MHz}, \mathrm{CDCl}_{3}\right)$ 8: 199.30, 195.90, $169.39,144.30,138.05,134.17,133.69,133.03,130.62,129.89,128.58,122.46$, $120.78,26.31,25.40$.

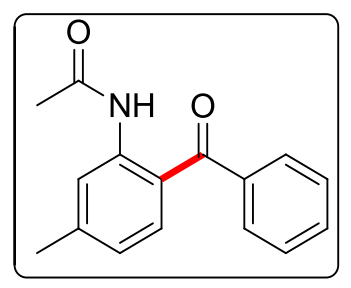

$N$-(2-Benzoyl-5-methylphenyl)acetamide $(\mathbf{3 j}):{ }^{[6]}$ Pale solid; $93.7 \mathrm{mg}, 74 \%$ yield; ${ }^{1} \mathrm{H}$ NMR (400 MHz, $\left.\mathrm{CDCl}_{3}\right) \delta: 11.01(\mathrm{~s}, 1 \mathrm{H}), 8.50(\mathrm{~s}, 1 \mathrm{H}), 7.66(\mathrm{~d}, J=7.2 \mathrm{~Hz}, 2 \mathrm{H})$, 7.59-7.56 (m, 1H), 7.49-7.43(m, 3H), $6.88(\mathrm{~d}, J=8.0 \mathrm{~Hz}, 1 \mathrm{H}), 2.42(\mathrm{~s}, 3 \mathrm{H}), 2.22(\mathrm{~s}$, $3 \mathrm{H}) ;{ }^{13} \mathrm{C} \mathrm{NMR}\left(100 \mathrm{MHz}, \mathrm{CDCl}_{3}\right) \delta: 199.49,169.12,145.70,140.74,138.92,133.78$, $132.07,129.60,128.16,122.82,121.58,120.45,25.24,22.05$.

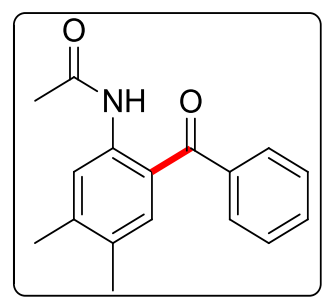

$N$-(2-Benzoyl-4,5-dimethylphenyl)acetamide (3k): ${ }^{[6]}$ White solid; $101.5 \mathrm{mg}, 76 \%$ yield; ${ }^{1} \mathrm{H}$ NMR $\left(400 \mathrm{MHz}, \mathrm{CDCl}_{3}\right) \delta: 10.84(\mathrm{~s}, 1 \mathrm{H}), 8.44(\mathrm{~s}, 1 \mathrm{H}), 7.68(\mathrm{~d}, J=7.2 \mathrm{~Hz}$, 2H), 7.61-7.57 (m, 1H), 7.50-7.47 (m, 2H), 7.29 (s, 1H), $2.34(\mathrm{~s}, 3 \mathrm{H}), 2.21(\mathrm{~s}, 3 \mathrm{H})$, 2.19 (s, 3H); ${ }^{13} \mathrm{C}$ NMR (100 MHz, $\left.\mathrm{CDCl}_{3}\right) \delta: 199.52,168.99,144.30,139.02,138.61$, 
$134.30,132.09,130.38,129.66,128.20,122.38,121.05,25.22,20.45,19.11$.<smiles>CC(=O)Nc1cc(Cl)cc(Cl)c1C(=O)c1ccccc1</smiles>

$N$-(2-Benzoyl-3,5-dichlorophenyl)acetamide (31): White solid; $115.1 \mathrm{mg}, 75 \%$ yield;

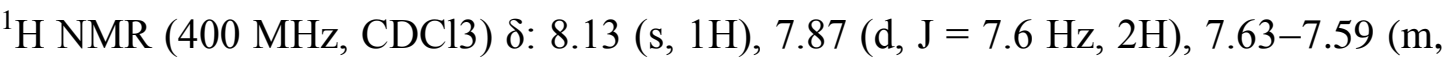
1H), 7.50-7.48 (m, 3H), 7.25 (s, 1H), $2.06(\mathrm{~s}, 3 \mathrm{H}) ;{ }^{13} \mathrm{C}$ NMR (100 MHz, CDCl3) $\delta$ : $193.58,168.43,136.71,135.77,133.50,131.31,131.03,130.95,130.61,130.40$, 128.42, 128.04, 23.31. HRMS (ESI) $\left([\mathrm{M}+\mathrm{H}]^{+}\right)$Calcd. For $\mathrm{C}_{15} \mathrm{H}_{12} \mathrm{C}_{12} \mathrm{NO}_{2}: 308.0245$, Found: 308.0249.

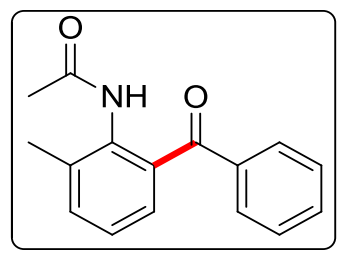

$N$-(2-Benzoyl-6-methylphenyl)acetamide $(\mathbf{3 m}):{ }^{[8]}$ Pale solid; $103.8 \mathrm{mg}, 82 \%$ yield; ${ }^{1} \mathrm{H}$ NMR (400 MHz, $\left.\mathrm{CDCl}_{3}\right) \delta: 11.02(\mathrm{~s}, 1 \mathrm{H}), 8.50(\mathrm{~s}, 1 \mathrm{H}), 7.67(\mathrm{~d}, J=7.6 \mathrm{~Hz}, 2 \mathrm{H})$, $7.59(\mathrm{t}, J=7.2 \mathrm{~Hz}, 1 \mathrm{H}), 7.50-7.44(\mathrm{~m}, 3 \mathrm{H}), 6.88(\mathrm{~d}, J=8.0 \mathrm{~Hz}, 1 \mathrm{H}), 2.43(\mathrm{~s}, 3 \mathrm{H})$, $2.23(\mathrm{~s}, 3 \mathrm{H}) ;{ }^{13} \mathrm{C}$ NMR $\left(100 \mathrm{MHz}, \mathrm{CDCl}_{3}\right) \delta: 199.61,169.22,145.81,140.80,138.99$, $133.87,132.16,129.67,128.23,122.89,121.65,120.50,25.34,22.13$.<smiles>O=C(CCc1ccccc1)Nc1c(F)cccc1C(=O)c1ccccc1</smiles>

$N$-(2-Benzoyl-6-fluorophenyl)acetamide (3n): White solid; $99.0 \mathrm{mg}, 77 \%$ yield; ${ }^{1} \mathrm{H}$ NMR (400 MHz, $\left.\mathrm{CDCl}_{3}\right) \delta: 8.35(\mathrm{~s}, 1 \mathrm{H}), 7.83(\mathrm{~d}, J=7.6 \mathrm{~Hz}, 2 \mathrm{H}), 7.62-7.58(\mathrm{~m}, 1 \mathrm{H})$, 7.49-7.45 (m, 2H), 7.28-7.26 (m, 1H), 7.24-7.22 (m, 2H), $2.08(\mathrm{~s}, 3 \mathrm{H}) ;{ }^{13} \mathrm{C}$ NMR 
$\left(100 \mathrm{MHz}, \mathrm{CDCl}_{3}\right) \delta: 195.64(\mathrm{~d}, J=2.4 \mathrm{~Hz}), 168.29,156.57(\mathrm{~d}, J=249.8 \mathrm{~Hz}), 136.85$, 134.22, 133.21, 130.35, 128.35, 125.92 (d, $J=3.5 \mathrm{~Hz}), 125.78$ (d, $J=7.9 \mathrm{~Hz}), 124.52$ $(\mathrm{d}, J=13.4 \mathrm{~Hz}), 119.12(\mathrm{~d}, J=20.9 \mathrm{~Hz}), 23.35$. HRMS (ESI) $\left([\mathrm{M}+\mathrm{H}]^{+}\right)$Calcd. For $\mathrm{C}_{15} \mathrm{H}_{13} \mathrm{FNO}_{2}$ : 258.0930, Found: 258.0934.<smiles>O=C(CCc1ccccc1)Nc1c(Cl)cccc1C(=O)c1ccccc1</smiles>

$N$-(2-Benzoyl-6-chlorophenyl)acetamide (3o): Pale solid; $103.8 \mathrm{mg}, 76 \%$ yield; ${ }^{1} \mathrm{H}$ NMR (400 MHz, $\left.\mathrm{CDCl}_{3}\right) \delta: 8.69(\mathrm{~s}, 1 \mathrm{H}), 7.88(\mathrm{~d}, J=7.2 \mathrm{~Hz}, 2 \mathrm{H}), 7.57-7.53(\mathrm{~m}, 1 \mathrm{H})$, $7.45-7.37(\mathrm{~m}, 3 \mathrm{H}), 7.23-7.21(\mathrm{~m}, 1 \mathrm{H}), 7.12(\mathrm{t}, J=7.6 \mathrm{~Hz}, 1 \mathrm{H}), 2.03(\mathrm{~s}, 3 \mathrm{H}) ;{ }^{13} \mathrm{C}$ NMR (100 MHz, $\left.\mathrm{CDCl}_{3}\right)$ \&: 194.95, 168.56, 136.22, 135.79, 132.94, 132.05, 131.57, 130.28, 129.76, 128.03, 127.89, 125.46, 23.01. HRMS (ESI) $\left([\mathrm{M}+\mathrm{H}]^{+}\right)$Calcd. For $\mathrm{C}_{15} \mathrm{H}_{13} \mathrm{ClNO}_{2}$ : 274.0635, Found: 274.0640 .

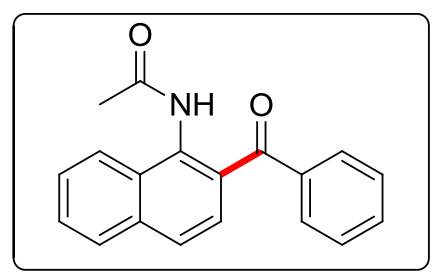

$N$-(2-Benzoylnaphthalen-1-yl)acetamide (3p): ${ }^{[8]}$ Pale solid; $98.3 \mathrm{mg}, 68 \%$ yield; ${ }^{1} \mathrm{H}$ NMR (400 MHz, $\left.\mathrm{CDCl}_{3}\right) \delta: 10.58(\mathrm{~s}, 1 \mathrm{H}), 9.03(\mathrm{~s}, 1 \mathrm{H}), 8.07$ (s, 1H), 7.87 (d, J=8.0 $\mathrm{Hz}, 1 \mathrm{H}), 7.79$ (d, $J=7.6 \mathrm{~Hz}, 2 \mathrm{H}), 7.72(\mathrm{~d}, J=8.4 \mathrm{~Hz}, 1 \mathrm{H}), 7.67-7.63(\mathrm{~m}, 1 \mathrm{H})$, 7.60-7.51 (m, 3H), 7.44-7.40 (m, 1H), $2.26(\mathrm{~s}, 3 \mathrm{H}) ;{ }^{13} \mathrm{C} \mathrm{NMR}\left(100 \mathrm{MHz}, \mathrm{CDCl}_{3}\right) \delta$ : $199.68,168.98$, 138.69, 136.06, 135.76, 135.43, 132.74, 130.16, 129.39, 128.88, 128.42 , 128.06, 127.73, 125.69, 124.16, 118.74, 25.25 . 


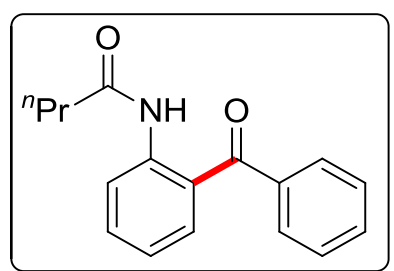

$N$-(2-Benzoylphenyl)butyramide (3q): ${ }^{[9]}$ White solid; $109.5 \mathrm{mg}, 82 \%$ yield; ${ }^{1} \mathrm{H}$ NMR (400 MHz, $\left.\mathrm{CDCl}_{3}\right) \delta: 10.87(\mathrm{~s}, 1 \mathrm{H}), 8.68(\mathrm{~d}, J=8.0 \mathrm{~Hz}, 1 \mathrm{H}), 7.70(\mathrm{~d}, J=7.2$ $\mathrm{Hz}, 2 \mathrm{H}), 7.61-7.55(\mathrm{~m}, 3 \mathrm{H}), 7.50-7.47(\mathrm{~m}, 2 \mathrm{H}), 7.09-7.05(\mathrm{~m}, 1 \mathrm{H}), 2.42(\mathrm{t}, J=7.6$ $\mathrm{Hz}, 2 \mathrm{H}), 1.84-1.75(\mathrm{~m}, 2 \mathrm{H}), 1.01(\mathrm{t}, J=7.6 \mathrm{~Hz}, 3 \mathrm{H}) ;{ }^{13} \mathrm{C} \mathrm{NMR}\left(100 \mathrm{MHz}, \mathrm{CDCl}_{3}\right) \delta$ : $199.75,172.19,140.71,138.75,134.28,133.57,132.37,129.81,128.28,123.09$, $121.85,121.46,40.39,18.94,13.70$.

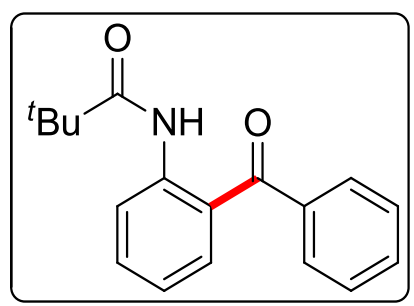

$N$-(2-Benzoylphenyl)pivalamide $(3 \mathrm{rr}):{ }^{[10]}$ White solid; $108.2 \mathrm{mg}, 77 \%$ yield; ${ }^{1} \mathrm{H}$ NMR (400 MHz, $\left.\mathrm{CDCl}_{3}\right) \delta: 11.24(\mathrm{~s}, 1 \mathrm{H}), 8.73(\mathrm{~d}, J=8.4 \mathrm{~Hz}, 1 \mathrm{H}), 7.69$ (d, $J=7.2$ $\mathrm{Hz}, 2 \mathrm{H}), 7.60-7.54(\mathrm{~m}, 3 \mathrm{H}), 7.50-7.46(\mathrm{~m}, 2 \mathrm{H}), 7.08-7.04(\mathrm{~m}, 1 \mathrm{H}), 1.36(\mathrm{~s}, 9 \mathrm{H}) ;{ }^{13} \mathrm{C}$ NMR (100 MHz, $\left.\mathrm{CDCl}_{3}\right) \delta: 199.77,177.91,141.13,138.78,134.29,133.72,132.19$, $129.71,128.18,122.98,121.66,121.26,40.14,27.48$.

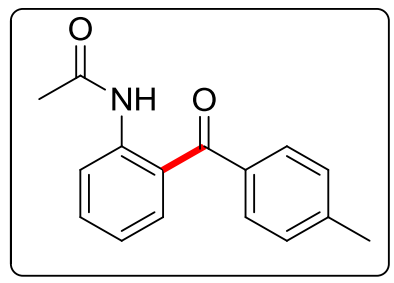

$N$-(2-(4-Methylbenzoyl)phenyl)acetamide (4a): ${ }^{[6]}$ White solid; $91.1 \mathrm{mg}, 72 \%$ yield; ${ }^{1} \mathrm{H}$ NMR $\left(400 \mathrm{MHz}, \mathrm{CDCl}_{3}\right) \delta: 10.72(\mathrm{~s}, 1 \mathrm{H}), 8.60(\mathrm{~d}, J=8.4 \mathrm{~Hz}, 1 \mathrm{H}), 7.62(\mathrm{~d}, J=$ $8.0 \mathrm{~Hz}, 2 \mathrm{H}), 7.55(\mathrm{~d}, J=7.6 \mathrm{~Hz}, 2 \mathrm{H}), 7.29(\mathrm{~d}, J=8.0 \mathrm{~Hz}, 2 \mathrm{H}), 7.10-7.06(\mathrm{~m}, 1 \mathrm{H})$, $2.45(\mathrm{~s}, 3 \mathrm{H}), 2.22(\mathrm{~s}, 3 \mathrm{H}) ;{ }^{13} \mathrm{C}$ NMR $\left(100 \mathrm{MHz}, \mathrm{CDCl}_{3}\right) \delta: 199.30,169.09,143.46$, $140.23,135.85,133.92,133.23,130.17,129.00,123.67,121.98,121.54,25.21,21.61$. 


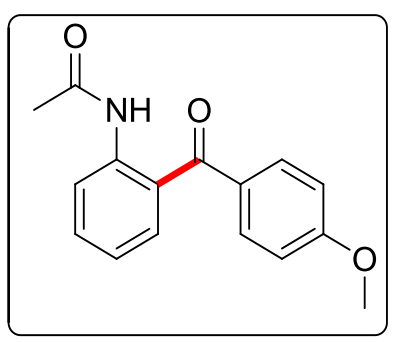

$N$-(2-(4-Methoxybenzoyl)phenyl)acetamide (4b): ${ }^{[6]}$ White solid; $94.2 \mathrm{mg}, 70 \%$ yield; ${ }^{1} \mathrm{H}$ NMR $\left(400 \mathrm{MHz}, \mathrm{CDCl}_{3}\right) \delta: 10.52(\mathrm{~s}, 1 \mathrm{H}), 8.56(\mathrm{~d}, J=8.4 \mathrm{~Hz}, 1 \mathrm{H}), 7.74(\mathrm{~d}$, $J=8.8 \mathrm{~Hz}, 2 \mathrm{H}), 7.56-7.52(\mathrm{~m}, 2 \mathrm{H}), 7.11-7.07(\mathrm{~m}, 1 \mathrm{H}), 6.97(\mathrm{~d}, J=8.4 \mathrm{~Hz}, 2 \mathrm{H}), 3.89$ (s, 3H), 2.20 (s, 3H); ${ }^{13} \mathrm{C}$ NMR (100 MHz, $\left.\mathrm{CDCl}_{3}\right) \delta: 197.85,168.95,163.40,139.79$, $133.46,132.67,132.54,130.87,124.21,121.99,121.67,113.60,55.48,25.10$.

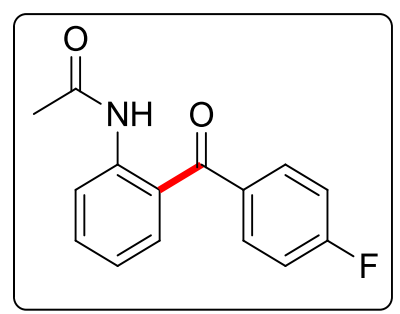

$N$-(2-(4-Fluorobenzoyl)phenyl)acetamide $(4 \mathrm{c}){ }^{[6]}$ Pale solid; $100.3 \mathrm{mg}, 78 \%$ yield; ${ }^{1} \mathrm{H}$ NMR (400 MHz, $\left.\mathrm{CDCl}_{3}\right) \delta: 10.63(\mathrm{~s}, 1 \mathrm{H}), 8.60(\mathrm{~d}, J=8.4 \mathrm{~Hz}, 1 \mathrm{H}), 7.77-7.73(\mathrm{~m}$, 2H), $7.59(\mathrm{t}, J=7.6 \mathrm{~Hz}, 1 \mathrm{H}), 7.52(\mathrm{~d}, J=7.6 \mathrm{~Hz}, 1 \mathrm{H}), 7.19-7.15(\mathrm{~m}, 2 \mathrm{H}), 7.11(\mathrm{t}, J=$ $7.6 \mathrm{~Hz}, 1 \mathrm{H}), 2.22(\mathrm{~s}, 3 \mathrm{H}) ;{ }^{13} \mathrm{C}$ NMR (100 MHz, $\left.\mathrm{CDCl}_{3}\right) \delta: 197.93,169.08,165.37(\mathrm{~d}$, $J=253.2 \mathrm{~Hz}), 140.25,134.71(\mathrm{~d}, J=3.1 \mathrm{~Hz}), 134.21,132.98,132.54(\mathrm{~d}, J=9.1 \mathrm{~Hz})$, $123.35,122.11,121.74,115.51(\mathrm{~d}, J=21.8 \mathrm{~Hz}), 25.15$.

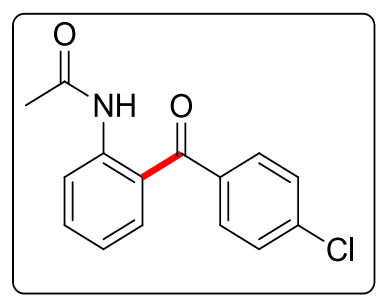

$N$-(2-(4-Chlorobenzoyl)phenyl)acetamide (4d): ${ }^{[6]}$ Pale solid; $110.6 \mathrm{mg}, 81 \%$ yield; ${ }^{1} \mathrm{H}$ NMR $\left(400 \mathrm{MHz}, \mathrm{CDCl}_{3}\right) \delta: 10.70(\mathrm{~s}, 1 \mathrm{H}), 8.62(\mathrm{~d}, J=8.4 \mathrm{~Hz}, 1 \mathrm{H}), 7.66(\mathrm{~d}, J=$ 8.4 Hz, 2H), 7.60-7.56 (m, 1H), 7.50-7.46 (m, 3H), 7.11-7.07 (m, 1H), $2.23(\mathrm{~s}, 3 \mathrm{H})$; 
${ }^{13} \mathrm{C}$ NMR (100 MHz, $\left.\mathrm{CDCl}_{3}\right) \delta: 198.26,169.09,140.45,139.00,136.87,134.44$, $133.11,131.27,128.65,123.00,122.11,121.69,25.21$.

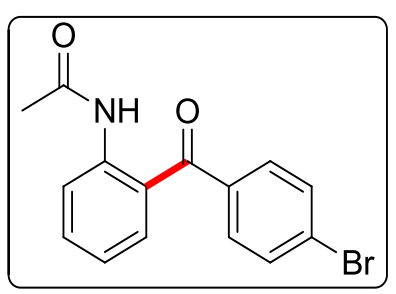

$N$-(2-(4-Bromobenzoyl)phenyl)acetamide $(4 \mathbf{e}):{ }^{[6]}$ White solid; $128.4 \mathrm{mg}, 81 \%$ yield;

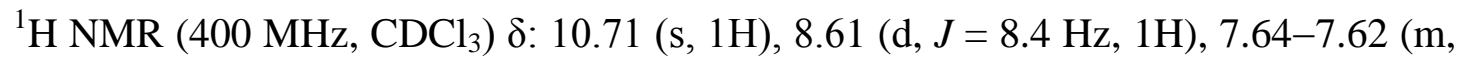
2H), 7.60-7.56 (m, 3H), 7.51-7.49 (m, 1H), $7.09(\mathrm{t}, J=7.6 \mathrm{~Hz}, 1 \mathrm{H}), 2.22(\mathrm{~s}, 3 \mathrm{H}),{ }^{13} \mathrm{C}$ NMR (100 MHz, $\left.\mathrm{CDCl}_{3}\right) \delta: 198.32,169.01,140.43,137.26,134.41,133.05,131.57$, $131.29,127.49,122.88,122.05,121.62,25.15$.

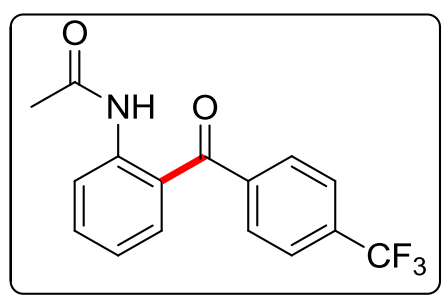

$N$-(2-(4-(Trifluoromethyl)benzoyl)phenyl)acetamide (4f): ${ }^{[6]}$ Pale solid; $109.0 \mathrm{mg}$, $71 \%$ yield; ${ }^{1} \mathrm{H}$ NMR (400 MHz, $\mathrm{CDCl}_{3}$ ) $\delta: 10.86$ (s, $\left.1 \mathrm{H}\right), 8.67(\mathrm{~d}, J=8.4 \mathrm{~Hz}, 1 \mathrm{H})$, 7.81-7.75 (m, 4H), 7.63-7.57 (m, 1H), 7.49 (d, $J=8.0 \mathrm{~Hz}, 1 \mathrm{H}), 7.10$ (t, $J=7.6 \mathrm{~Hz}$, 1H), $2.25(\mathrm{~s}, 3 \mathrm{H}) ;{ }^{13} \mathrm{C} \mathrm{NMR}\left(100 \mathrm{MHz}, \mathrm{CDCl}_{3}\right) \delta: 198.61,169.22,141.87\left(\mathrm{q}, J_{l}=1.3\right.$ $\left.\mathrm{Hz}, J_{2}=2.6 \mathrm{~Hz}\right), 140.93,135.02,133.76(\mathrm{~d}, J=32.6 \mathrm{~Hz}), 133.51,129.88,126.24(\mathrm{~d}$, $J=3.8 \mathrm{~Hz}), 125.38\left(\mathrm{q}, J_{1}=3.8 \mathrm{~Hz}, J_{2}=7.5 \mathrm{~Hz}\right), 122.37,122.18,121.65,25.27$.

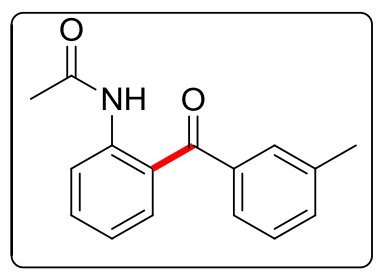

$N$-(2-(3-Methylbenzoyl)phenyl)acetamide (4g): ${ }^{[7]}$ White solid; $106.3 \mathrm{mg}, 84 \%$ yield; 
${ }^{1} \mathrm{H}$ NMR $\left(400 \mathrm{MHz}, \mathrm{CDCl}_{3}\right) \delta: 10.80(\mathrm{~s}, 1 \mathrm{H}), 8.62(\mathrm{~d}, J=8.0 \mathrm{~Hz}, 1 \mathrm{H}), 7.58-7.52(\mathrm{~m}$, 3H), 7.48-7.46 (m, 1H), 7.42-7.34 (m, 2H), 7.09 (t, $J=7.6 \mathrm{~Hz}, 1 \mathrm{H}), 2.42(\mathrm{~s}, 3 \mathrm{H})$, 2.22 (s, $3 \mathrm{H}) ;{ }^{13} \mathrm{C} \mathrm{NMR}\left(100 \mathrm{MHz}, \mathrm{CDCl}_{3}\right) \delta: 199.92,169.19,140.35,138.62,138.22$, 134.12, 133.45, 133.24, 130.23, 128.11, 127.13, 123.40, 122.01, 121.47, 25.19, 21.28.

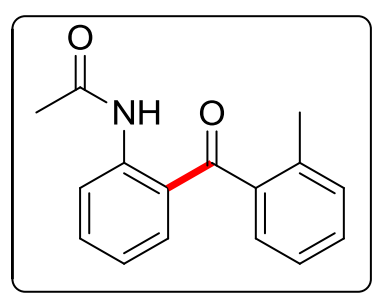

$N$-(2-(2-Methylbenzoyl)phenyl)acetamide $(\mathbf{4 h}):{ }^{[6]}$ White solid; $107.6 \mathrm{mg}, 85 \%$ yield; ${ }^{1} \mathrm{H}$ NMR (400 MHz, $\left.\mathrm{CDCl}_{3}\right) \delta: 11.55(\mathrm{~s}, 1 \mathrm{H}), 8.76(\mathrm{~d}, J=8.4 \mathrm{~Hz}, 1 \mathrm{H}), 7.58-7.54(\mathrm{~m}$, 1H), 7.40-7.38 (m, 2H), 7.30-7.24 (m, 3H), 7.01 (t, $J=7.6 \mathrm{~Hz}, 1 \mathrm{H}), 2.29$ (s, 3H), 2.28 (s, $3 \mathrm{H}) ;{ }^{13} \mathrm{C}$ NMR $\left(100 \mathrm{MHz}, \mathrm{CDCl}_{3}\right) \delta: 202.83,169.44,141.53,139.28,135.85$, 135.23, 134.43, 130.88, 130.18, 127.82, 125.32, 122.44, 122.08, 120.70, 25.47, 19.66.

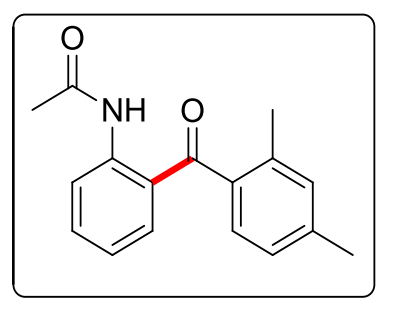

$N$-(2-(2,4-Dimethylbenzoyl)phenyl)acetamide (4i): ${ }^{[6]}$ White solid; $108.2 \mathrm{mg}, 81 \%$ yield; ${ }^{1} \mathrm{H}$ NMR $\left(400 \mathrm{MHz}, \mathrm{CDCl}_{3}\right) \delta: 11.47(\mathrm{~s}, 1 \mathrm{H}), 8.73(\mathrm{~d}, J=8.4 \mathrm{~Hz}, 1 \mathrm{H}), 7.57-$ 7.53(m, 1H), $7.41(\mathrm{~d}, J=8.0 \mathrm{~Hz}, 1 \mathrm{H}), 7.15-7.11(\mathrm{~m}, 2 \mathrm{H}), 7.07-7.05(\mathrm{~m}, 1 \mathrm{H}), 7.01(\mathrm{t}$, $J=7.6 \mathrm{~Hz}, 1 \mathrm{H}), 2.39$ (s, 3H), $2.28(\mathrm{~s}, 3 \mathrm{H}), 2.27$ (s, 3H); ${ }^{13} \mathrm{C} \mathrm{NMR}\left(100 \mathrm{MHz}, \mathrm{CDCl}_{3}\right)$ $\delta: 202.73,169.41,141.29,140.62,136.39,136.33,134.92,134.32,131.73,128.50$, $125.92,122.96,122.03,120.75,25.44,21.30,19.75$.

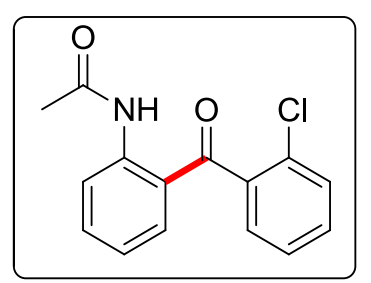


$N$-(2-(2-Chlorobenzoyl)phenyl)acetamide $(\mathbf{4 j}){ }^{[6]}$ Pale solid; $106.5 \mathrm{mg}, 78 \%$ yield;

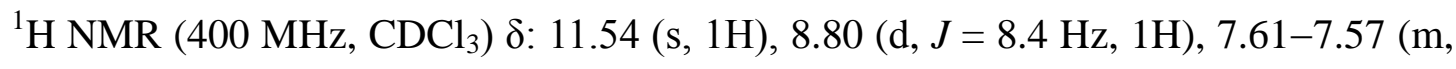
$1 \mathrm{H}), 7.49-7.45(\mathrm{~m}, 2 \mathrm{H}), 7.38-7.31(\mathrm{~m}, 3 \mathrm{H}), 7.01(\mathrm{t}, J=7.6 \mathrm{~Hz}, 1 \mathrm{H}), 2.30(\mathrm{~s}, 3 \mathrm{H}) ;{ }^{13} \mathrm{C}$ NMR (100 MHz, $\left.\mathrm{CDCl}_{3}\right)$ \&: 199.19, 169.53, 141.92, 138.81, 135.87, 134.52, 131.14, $130.93,130.10,128.63,126.70,122.21,121.28,120.63,25.56$.

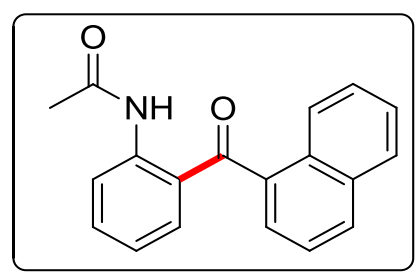

$N$-(2-(1-Naphthoyl)phenyl)acetamide (4k): ${ }^{[6]}$ White solid; $109.9 \mathrm{mg}, 76 \%$ yield; ${ }^{1} \mathrm{H}$ NMR (400 MHz, $\left.\mathrm{CDCl}_{3}\right) \delta: 11.58(\mathrm{~s}, 1 \mathrm{H}), 8.79(\mathrm{~d}, J=8.4 \mathrm{~Hz}, 1 \mathrm{H}), 7.98(\mathrm{~d}, J=7.2$ Hz, 1H), 7.94-7.90 (m, 2H), 7.57-7.48 (m, 5H), 7.40 (d, J= 7.2 Hz, 1H), 6.94-6.90 (m, 1H), 2.29 (s, 3H); ${ }^{13} \mathrm{C} \mathrm{NMR}\left(100 \mathrm{MHz}, \mathrm{CDCl}_{3}\right) \delta: 201.98,169.38,141.48,136.95$, $135.21,134.65,133.52,131.04,130.53,128.41,127.25,127.00,126.48,125.21$, $124.29,123.14,122.01,120.74,25.39$.

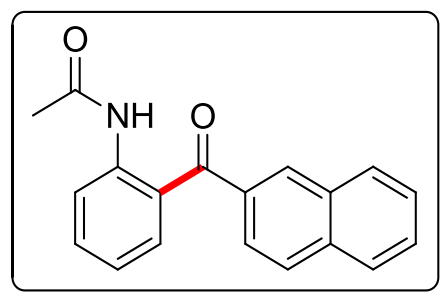

$N$-(2-(2-Naphthoyl)phenyl)acetamide (4l): ${ }^{[6]}$ White solid; $117.1 \mathrm{mg}, 81 \%$ yield; ${ }^{1} \mathrm{H}$ NMR (400 MHz, $\left.\mathrm{CDCl}_{3}\right) \delta: 10.76(\mathrm{~s}, 1 \mathrm{H}), 8.64$ (d, $\left.J=8.4 \mathrm{~Hz}, 1 \mathrm{H}\right), 8.14(\mathrm{~s}, 1 \mathrm{H})$, 7.92-7.87 (m, 3H), 7.82-7.80 (m, 1H), 7.61-7.52 (m, 4H), 7.07 (t, J = 7.6 Hz, 1H), $2.21(\mathrm{~s}, 3 \mathrm{H}) ;{ }^{13} \mathrm{C}$ NMR $\left(100 \mathrm{MHz}, \mathrm{CDCl}_{3}\right) \delta: 199.25,168.95,140.26,135.63,135.03$, $133.95,133.25,131.97,131.53,129.24,128.34,128.18,127.66,126.84,125.39$, $123.56,121.97,121.50,25.05$ 


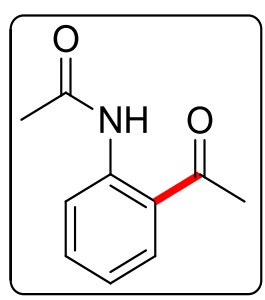

$N$-(2-Acetylphenyl)acetamide $(\mathbf{4 m}):^{[6]}$ White solid; $54.9 \mathrm{mg}, 62 \%$ yield; ${ }^{1} \mathrm{H}$ NMR $\left(400 \mathrm{MHz}, \mathrm{CDCl}_{3}\right) \delta: 11.69(\mathrm{~s}, 1 \mathrm{H}), 8.74(\mathrm{~d}, J=7.6 \mathrm{~Hz}, 1 \mathrm{H}), 7.89$ (d, $\left.J=6.8 \mathrm{~Hz}, 1 \mathrm{H}\right)$, 7.57-7.53 (m, 1H), $7.11(\mathrm{t}, J=7.2 \mathrm{~Hz}, 1 \mathrm{H}), 2.66(\mathrm{~s}, 3 \mathrm{H}), 2.23(\mathrm{~s}, 3 \mathrm{H}) ;{ }^{13} \mathrm{C}$ NMR $(100$ $\left.\mathrm{MHz}, \mathrm{CDCl}_{3}\right) \delta: 202.77,169.42,141.01,135.09,131.53,122.24,121.69,120.69$, $28.53,25.49$.

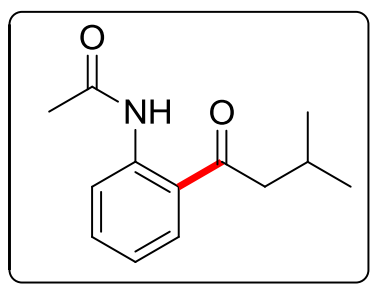

$N$-(2-(3-Methylbutanoyl)phenyl)acetamide (4n): ${ }^{[1]}$ White solid; $55.9 \mathrm{mg}, 51 \%$ yield; ${ }^{1} \mathrm{H}$ NMR $\left(400 \mathrm{MHz}, \mathrm{CDCl}_{3}\right) \delta: 11.75(\mathrm{~s}, 1 \mathrm{H}), 8.73(\mathrm{~d}, J=8.4 \mathrm{~Hz}, 1 \mathrm{H}), 7.90(\mathrm{~d}, J$ $=7.6 \mathrm{~Hz}, 1 \mathrm{H}), 7.55-7.52(\mathrm{~m}, 1 \mathrm{H}), 7.10(\mathrm{t}, J=7.6 \mathrm{~Hz}, 1 \mathrm{H}), 2.88(\mathrm{~d}, J=6.8 \mathrm{~Hz}, 2 \mathrm{H})$, 2.31-2.26 (m, 1H), $2.24(\mathrm{~s}, 3 \mathrm{H}), 1.02(\mathrm{~s}, 3 \mathrm{H}), 1.00(\mathrm{~s}, 3 \mathrm{H}) ;{ }^{13} \mathrm{C}$ NMR (100 MHz, $\left.\mathrm{CDCl}_{3}\right) \delta: 204.90,169.42,140.97,134.76,130.79,122.18,121.86,120.83,48.45$, $25.49,25.47,22.67$. 


\section{5. ${ }^{1} \mathrm{H}$ and ${ }^{13} \mathrm{C}$ NMR spectra of the products}
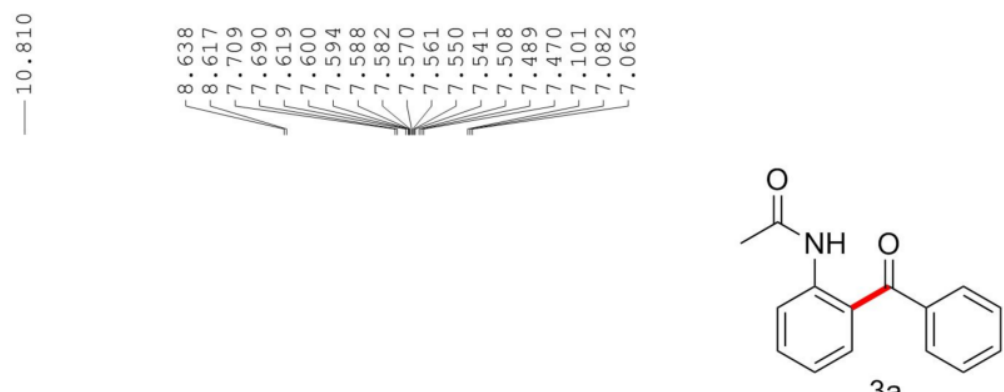

3a

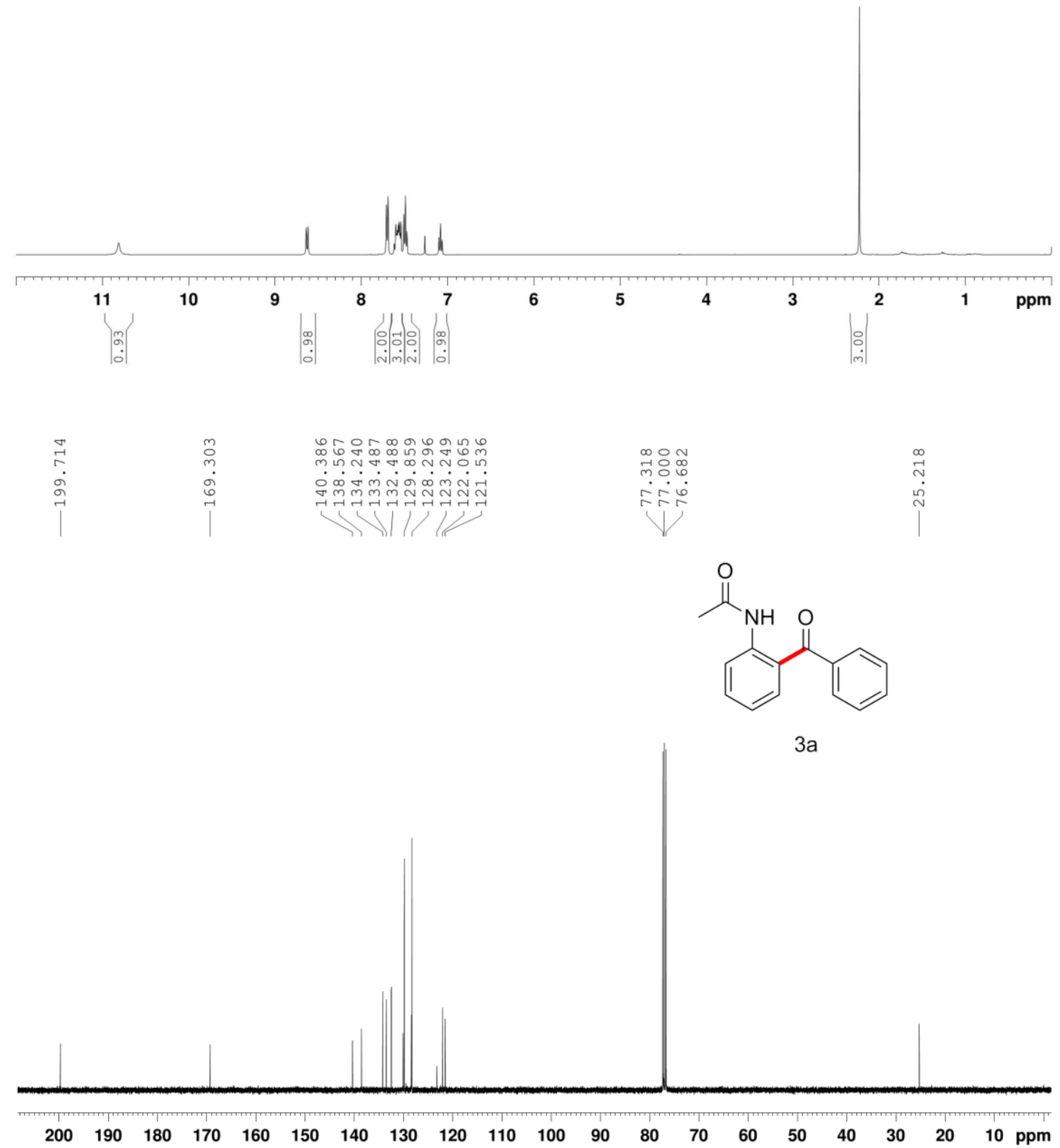




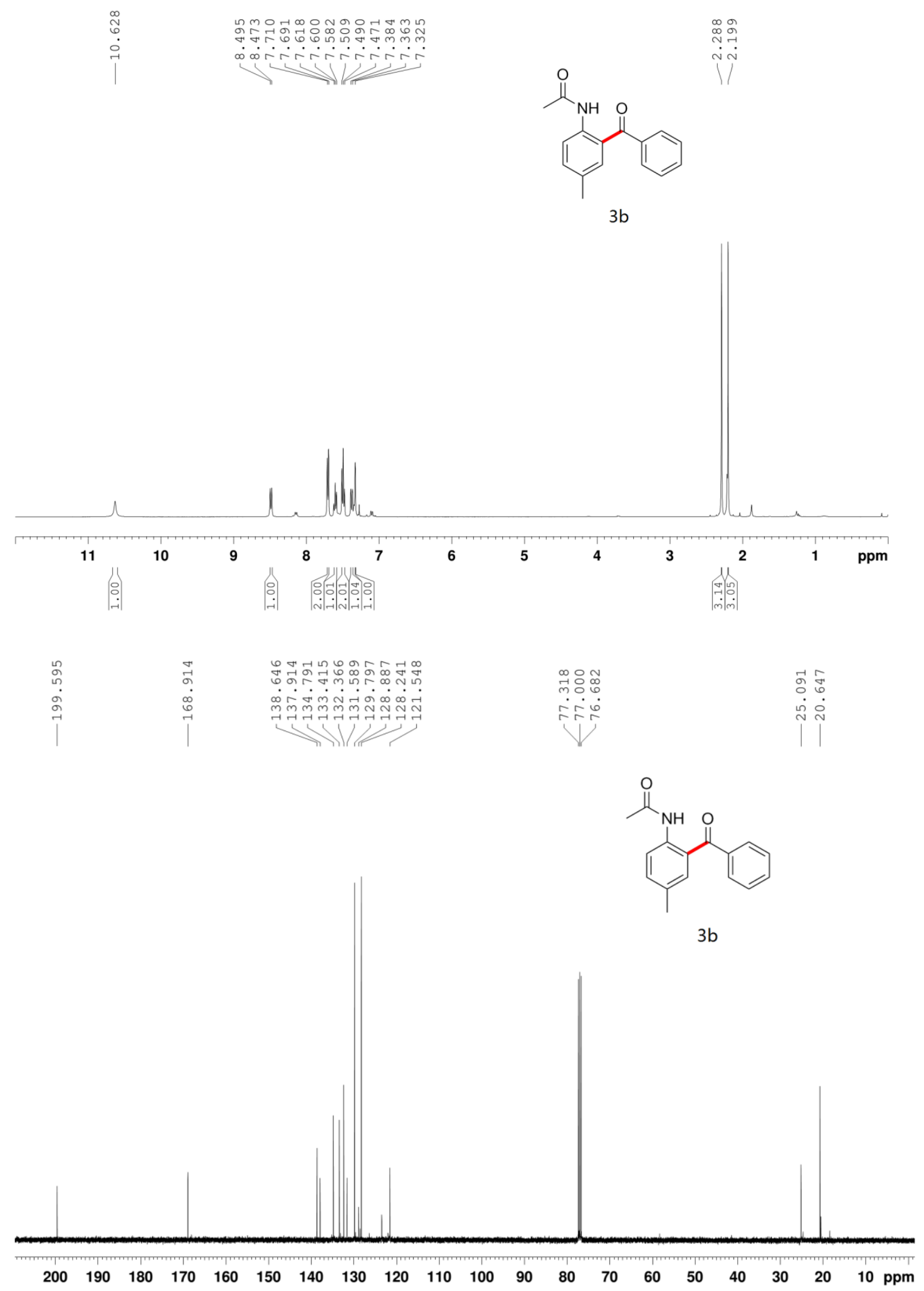




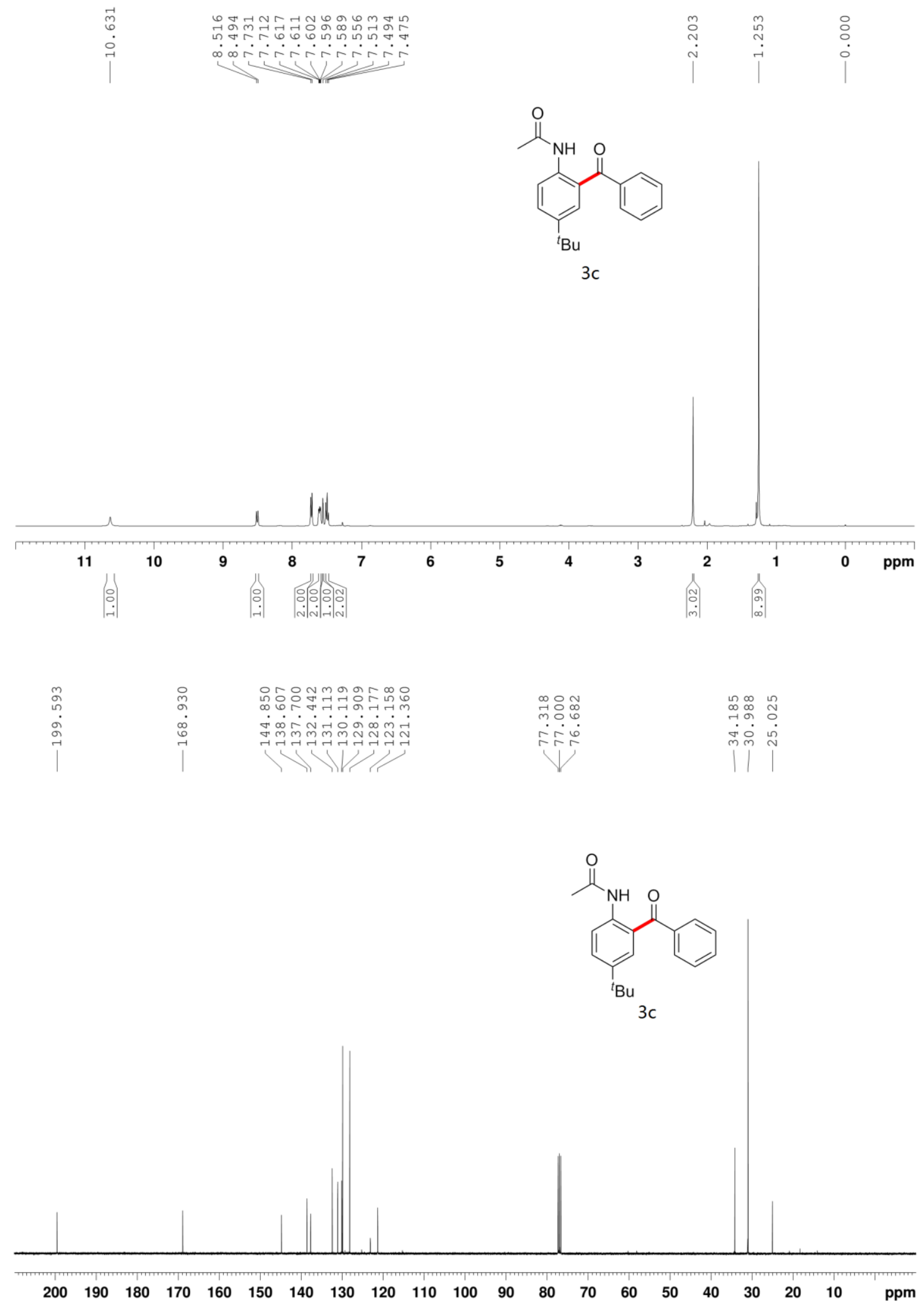



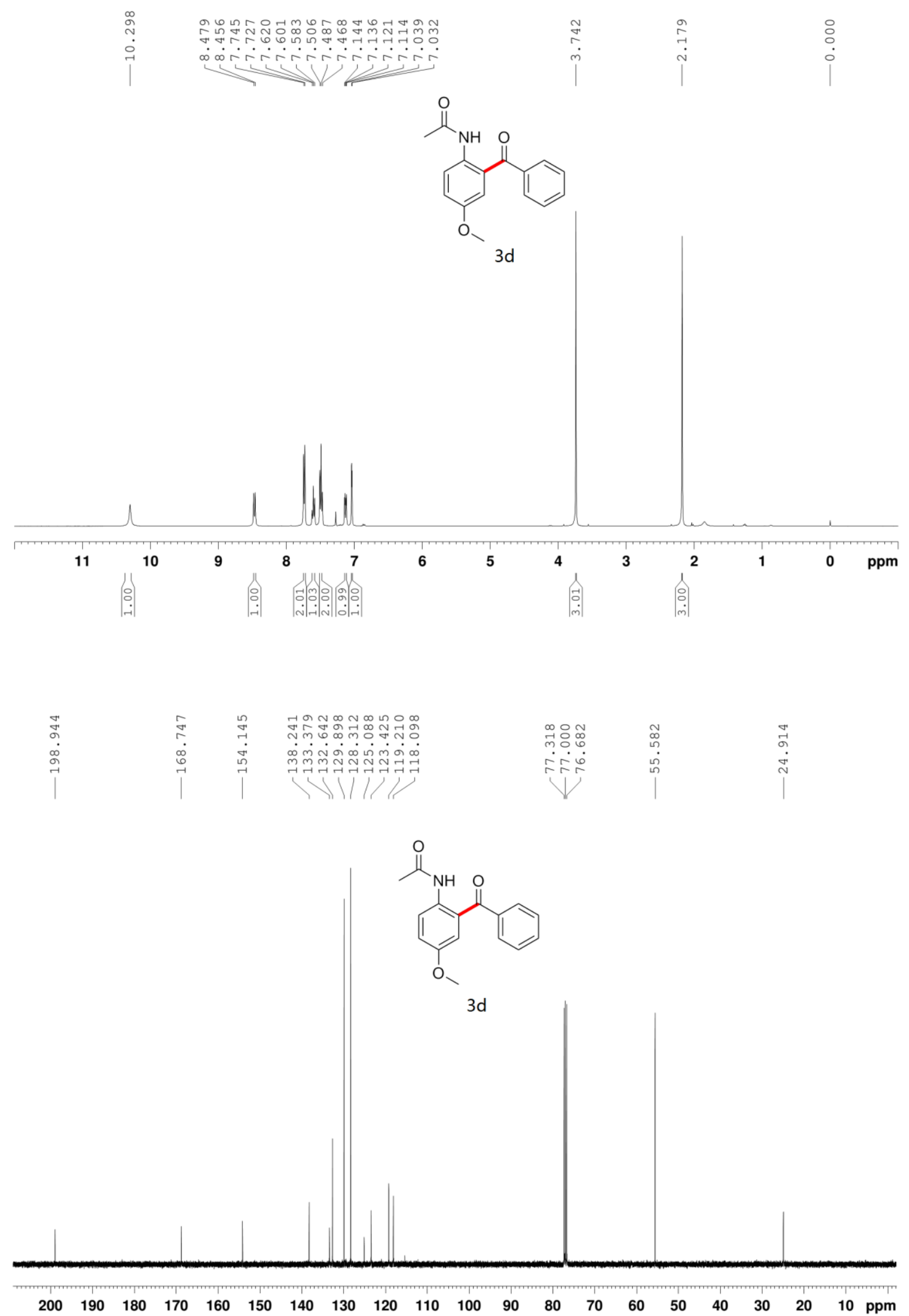

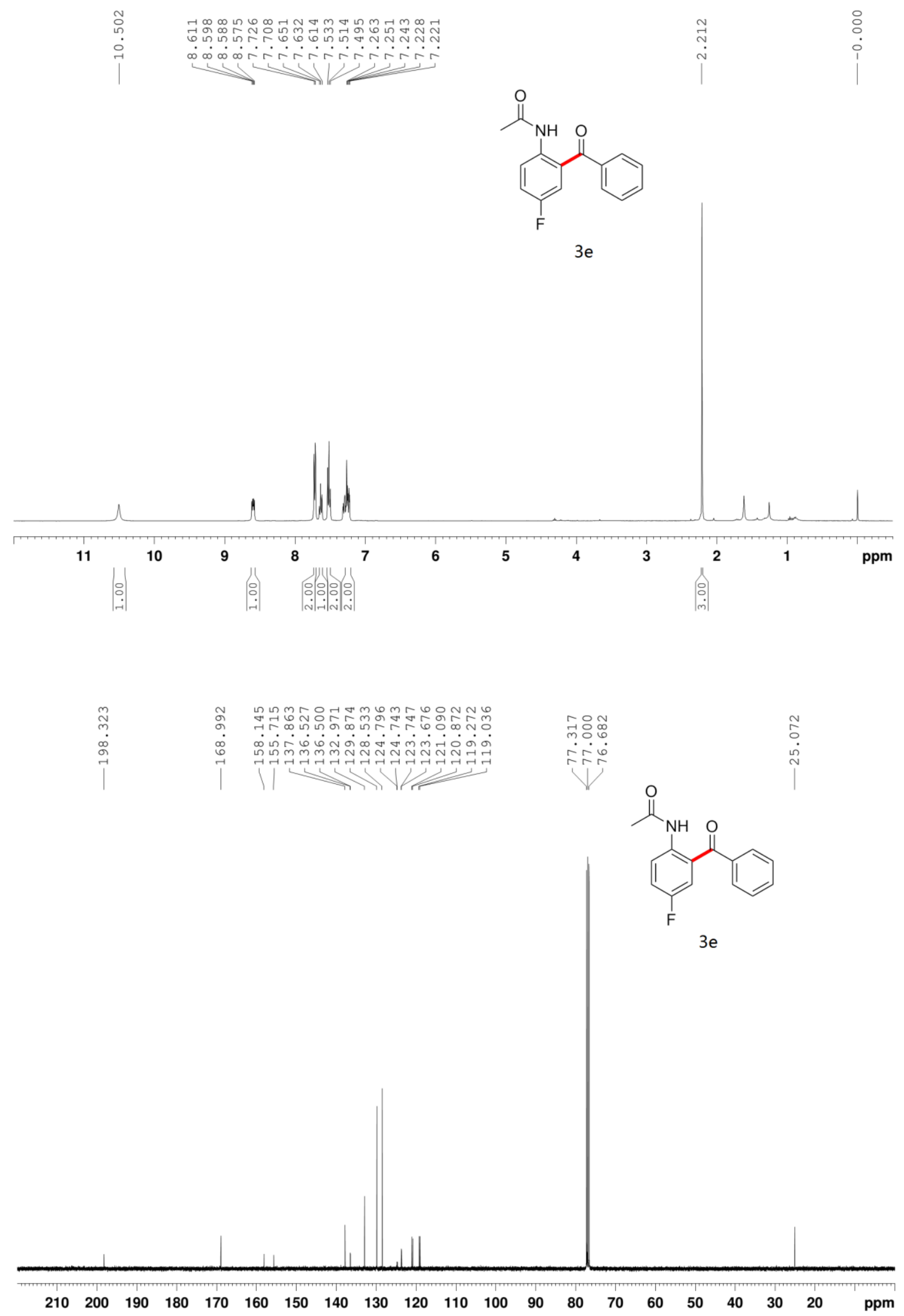


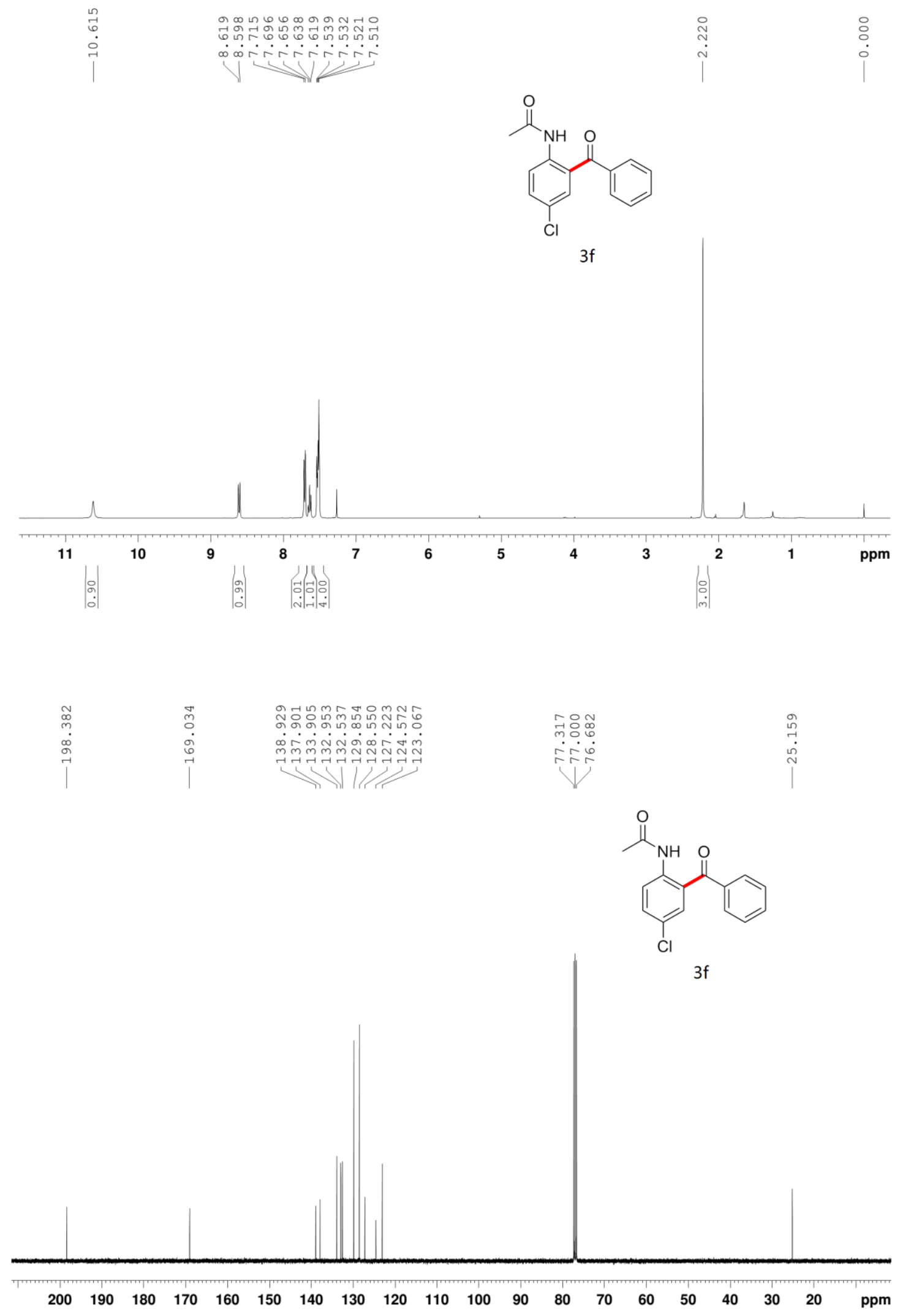




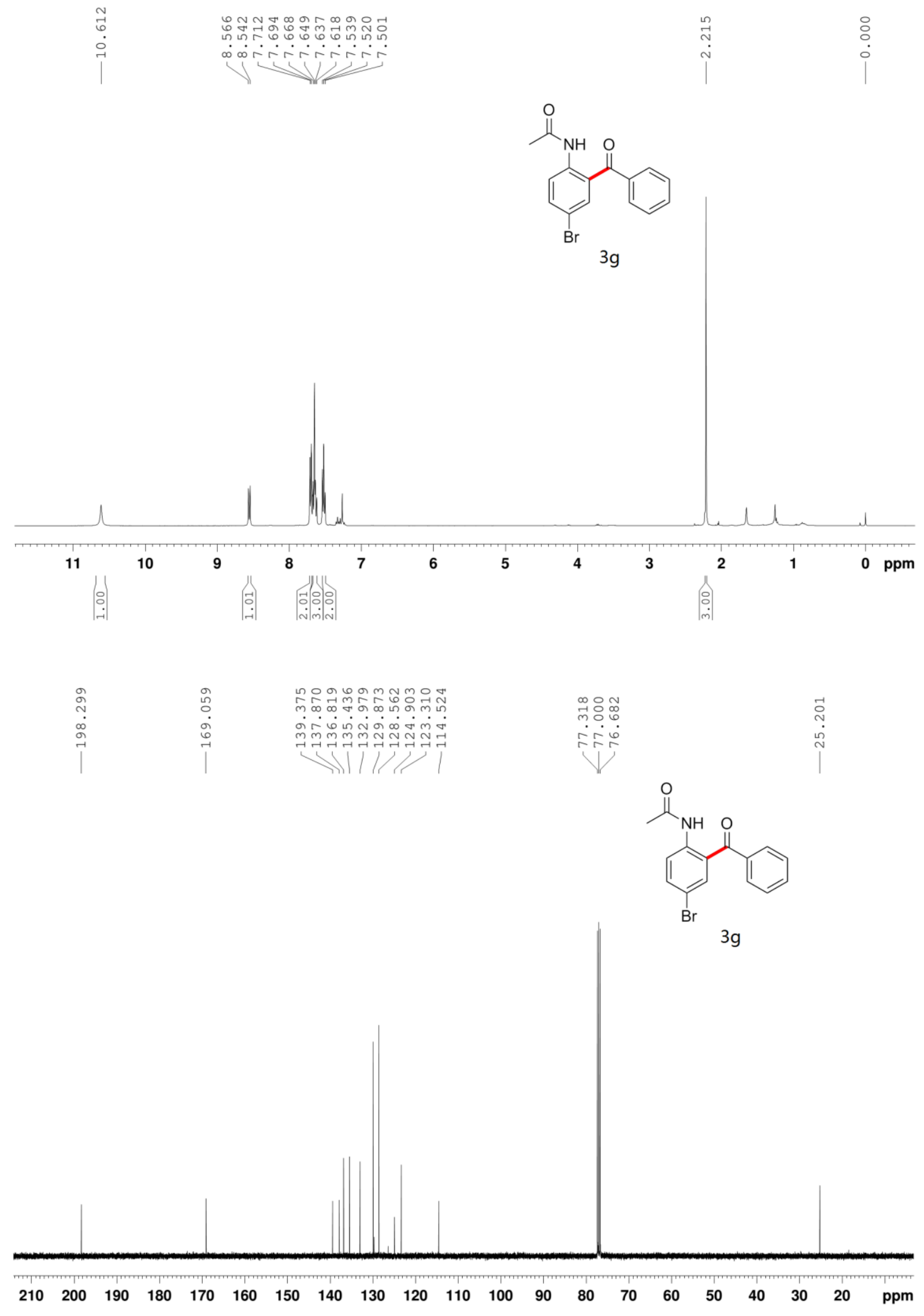



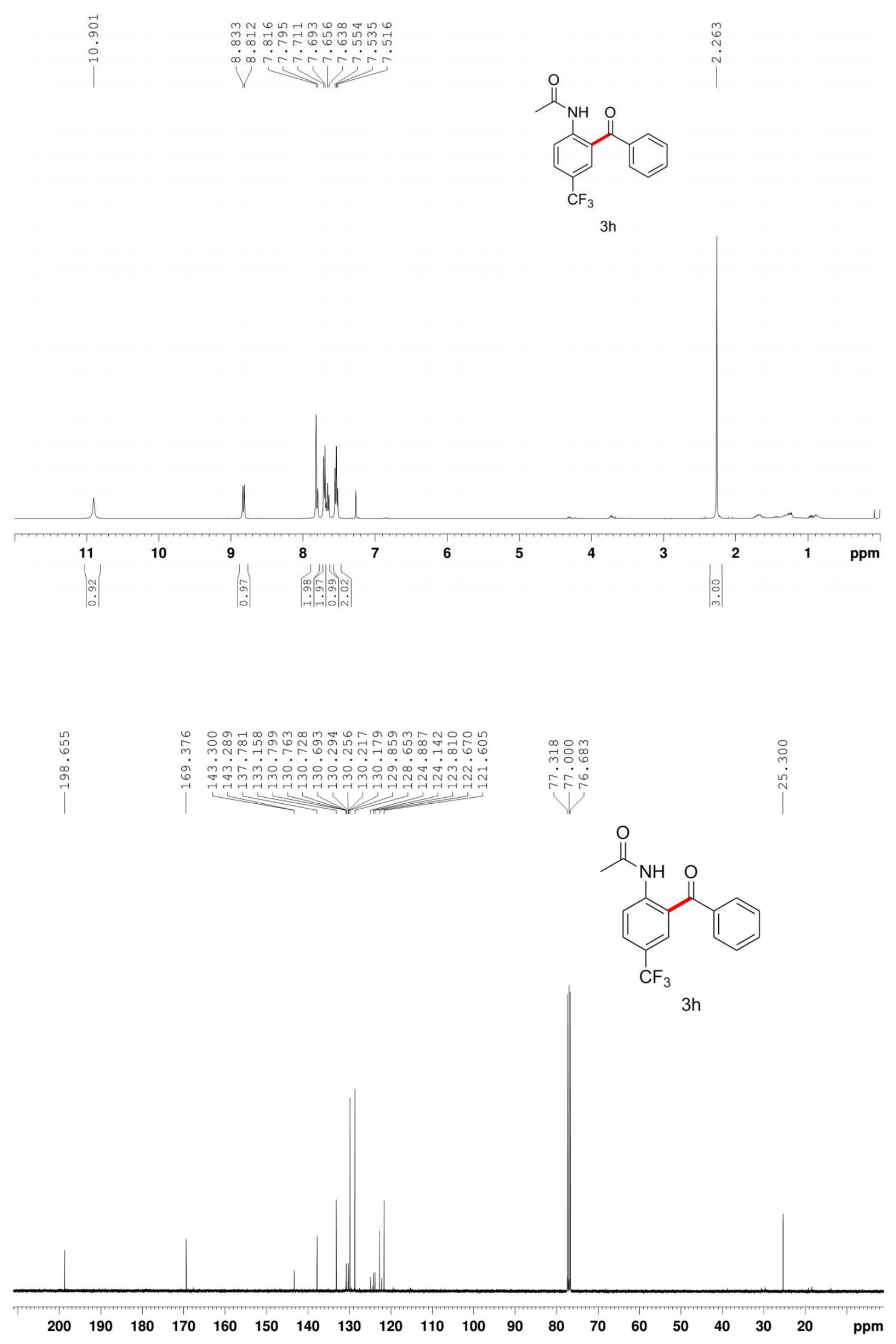

26 

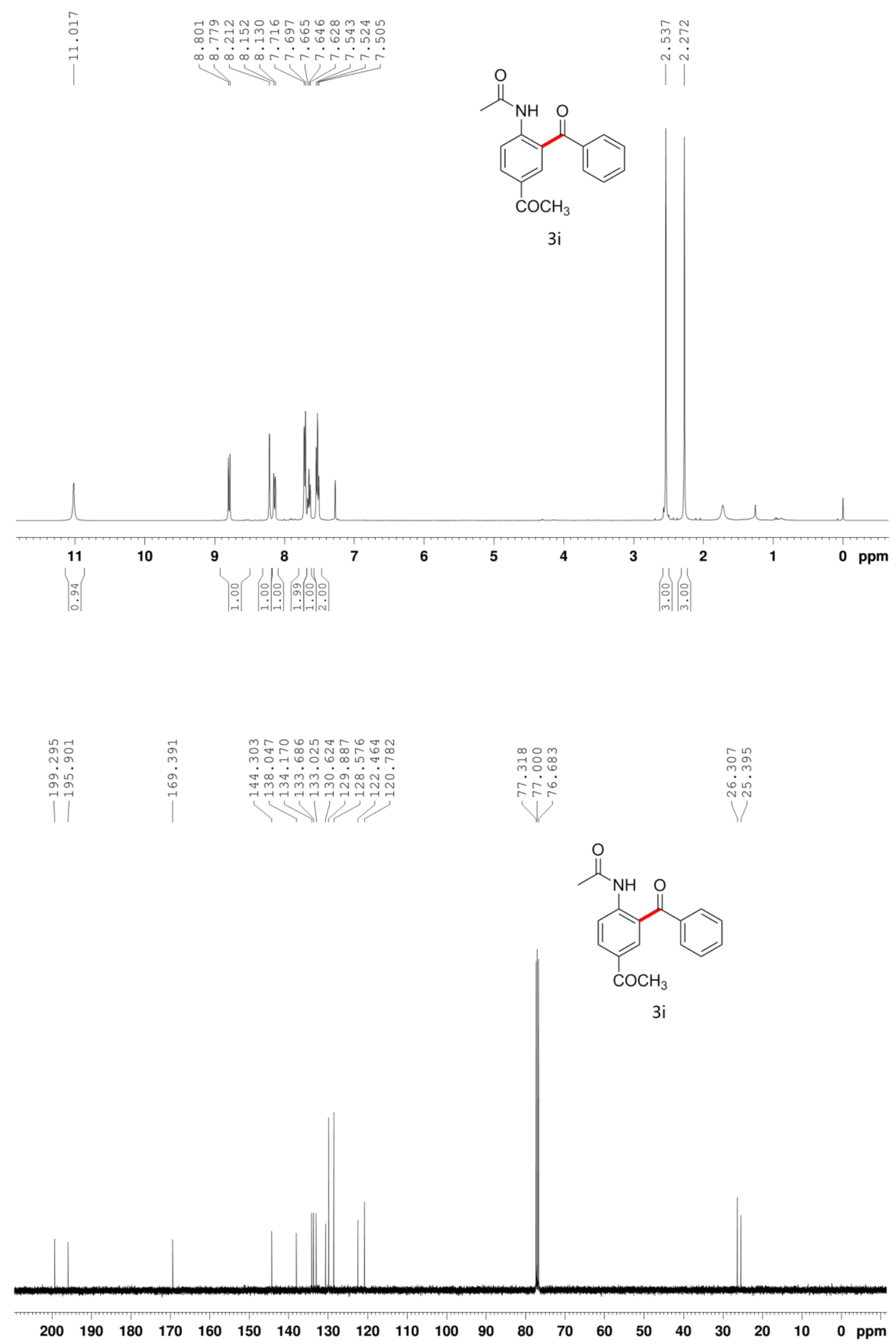


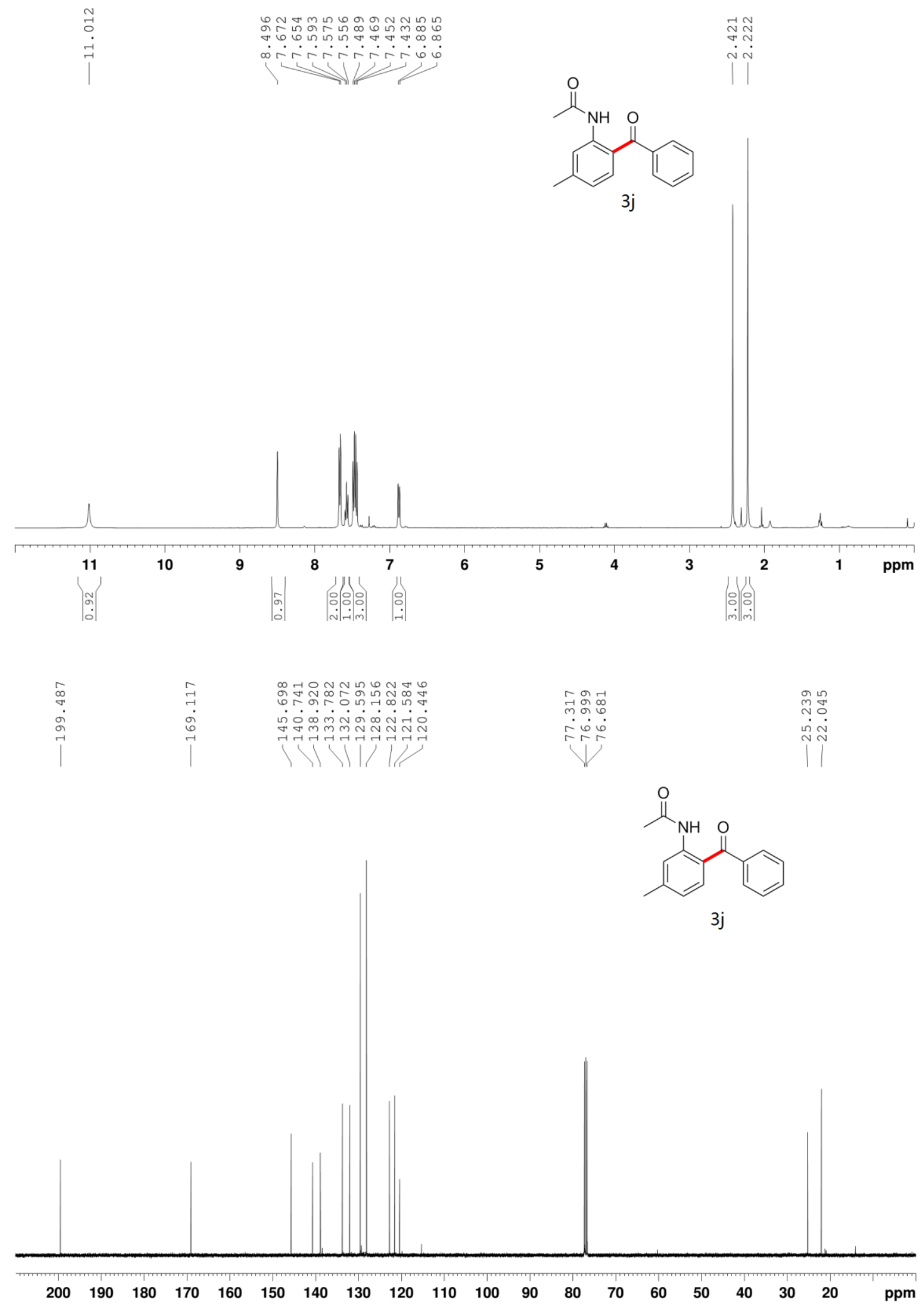




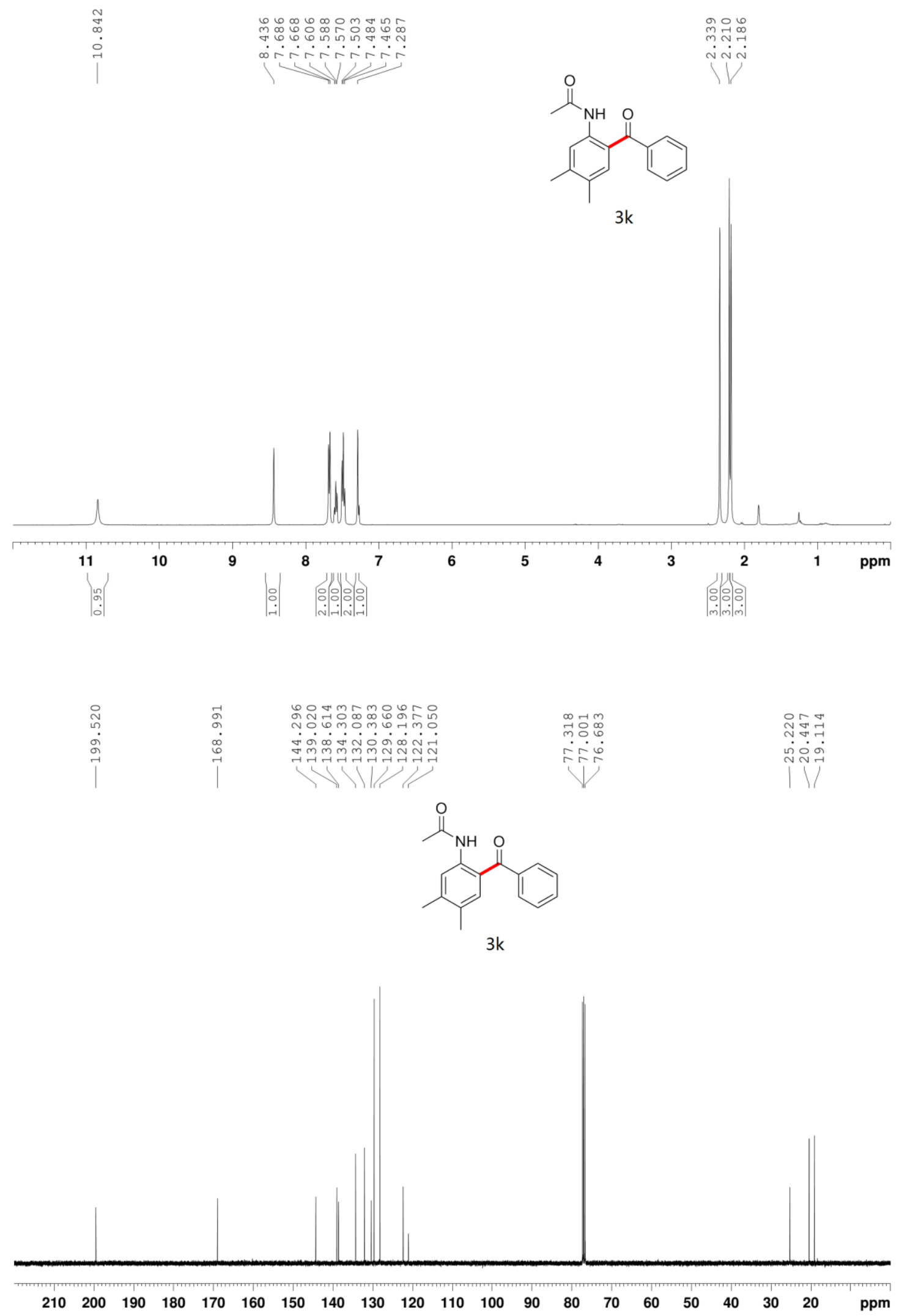




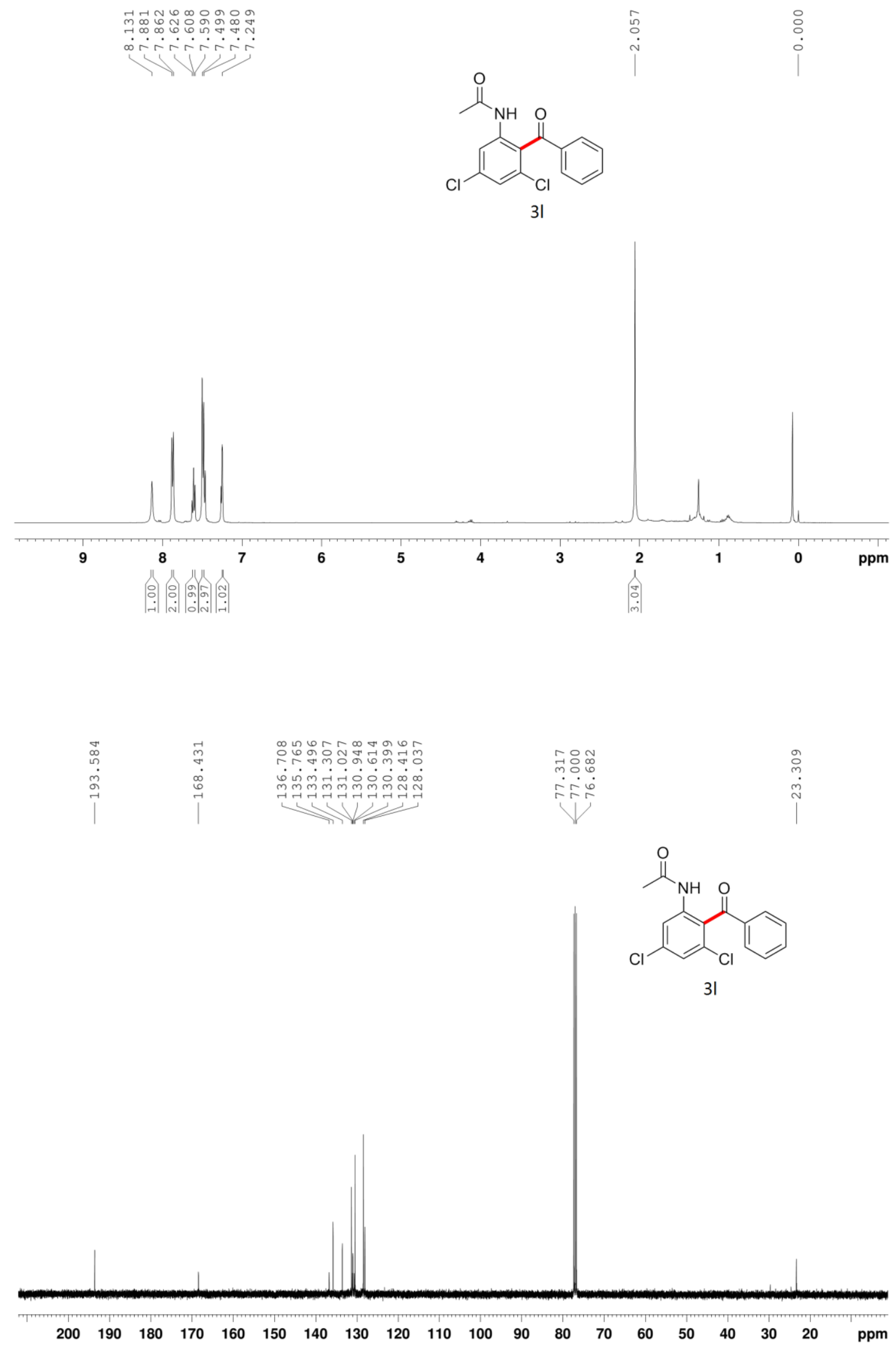




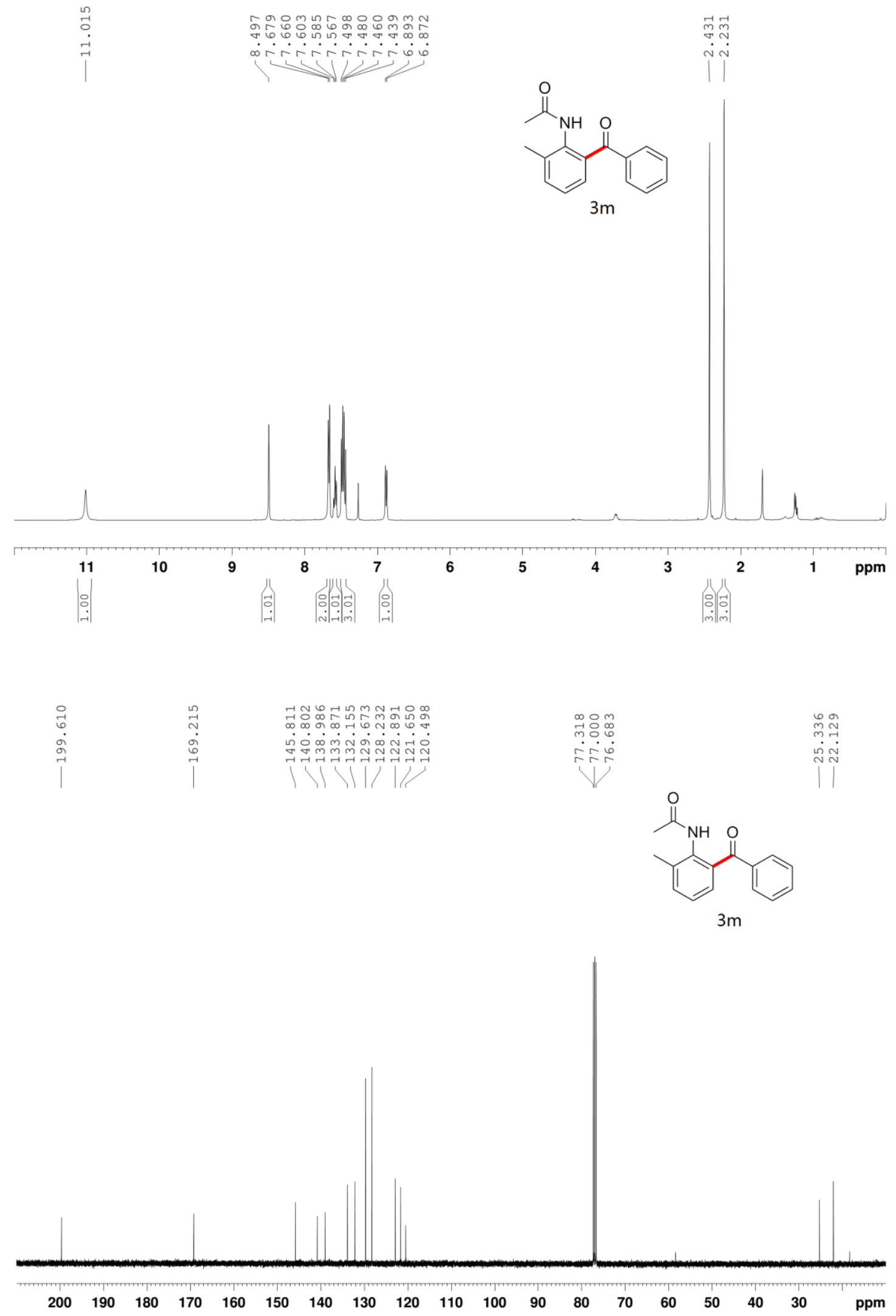



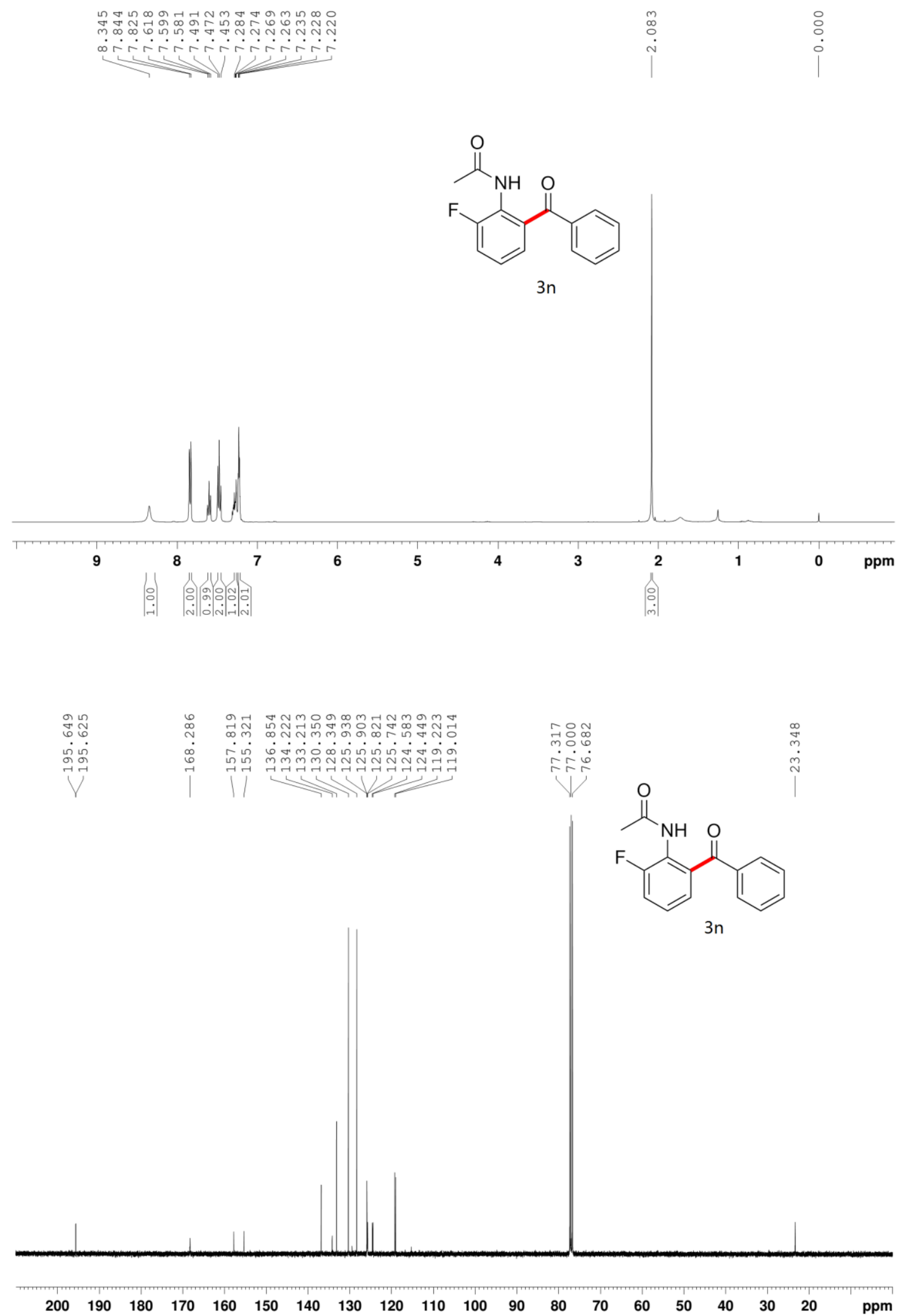


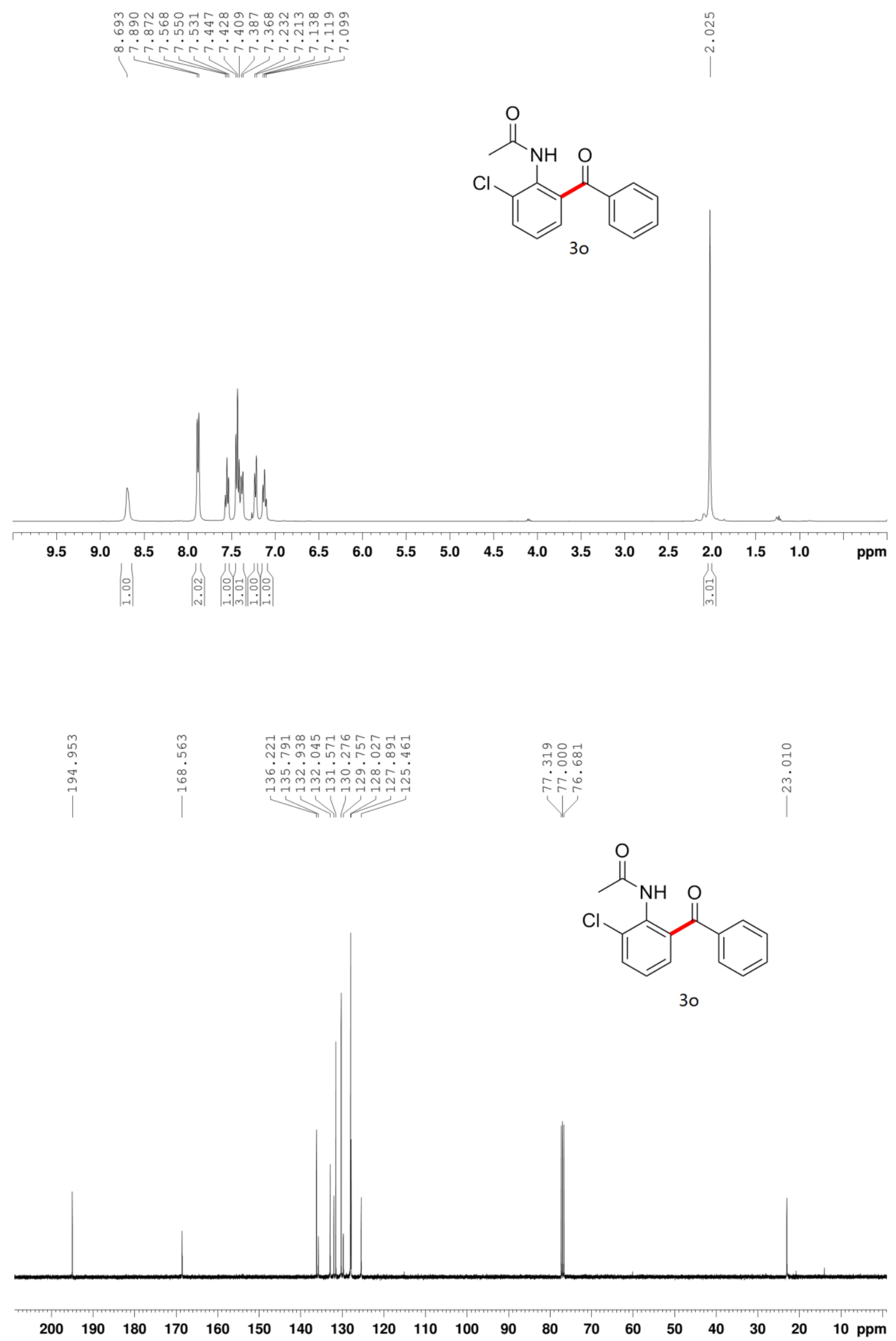



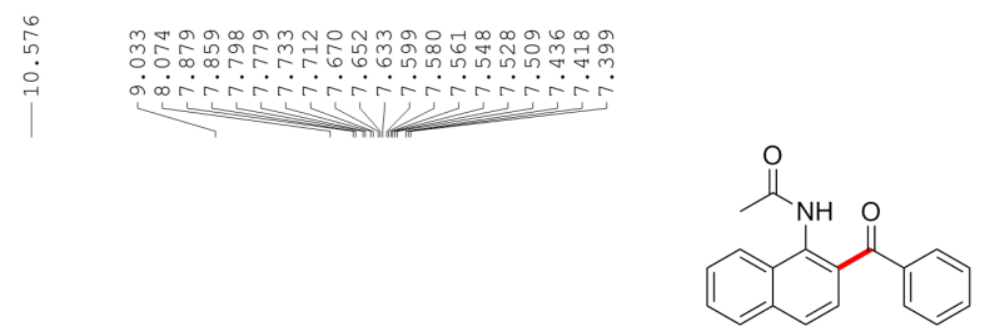

$3 p$
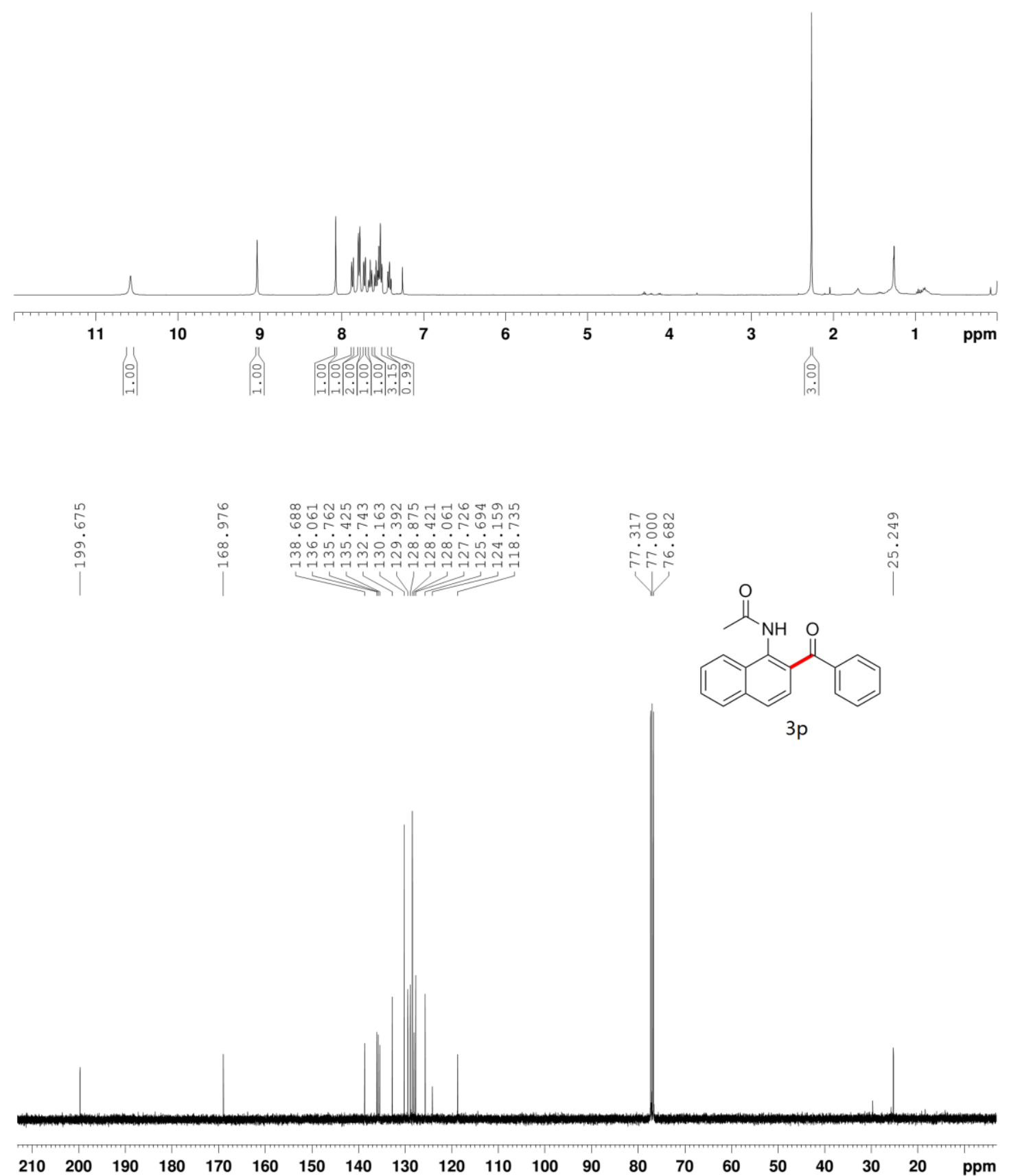


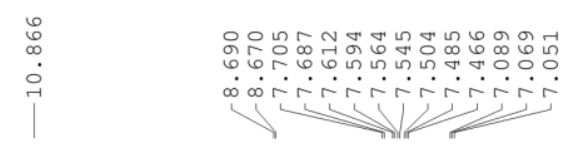<smiles>CC(C)C(=O)Nc1ccccc1C(=O)c1ccccc1</smiles>
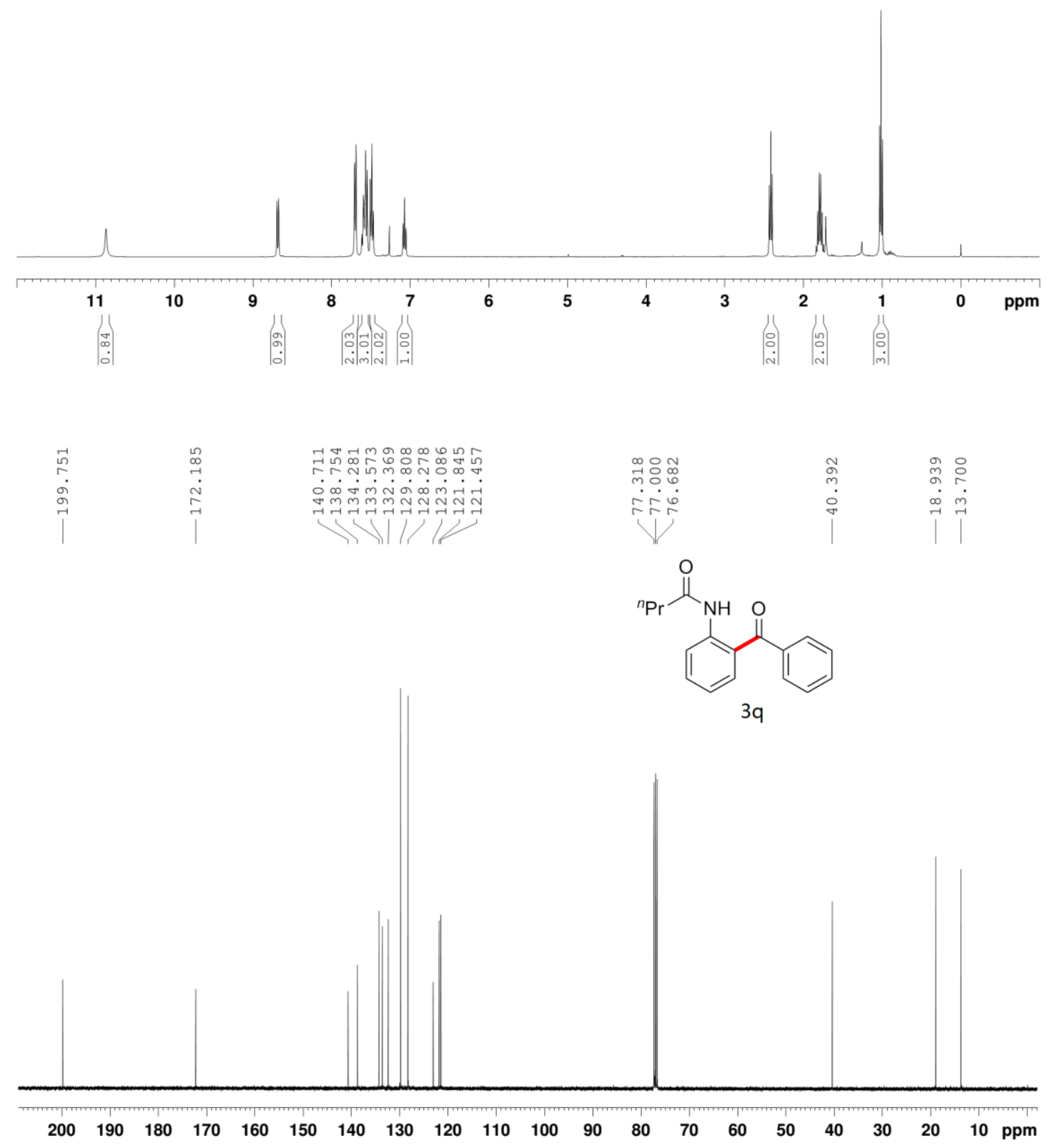

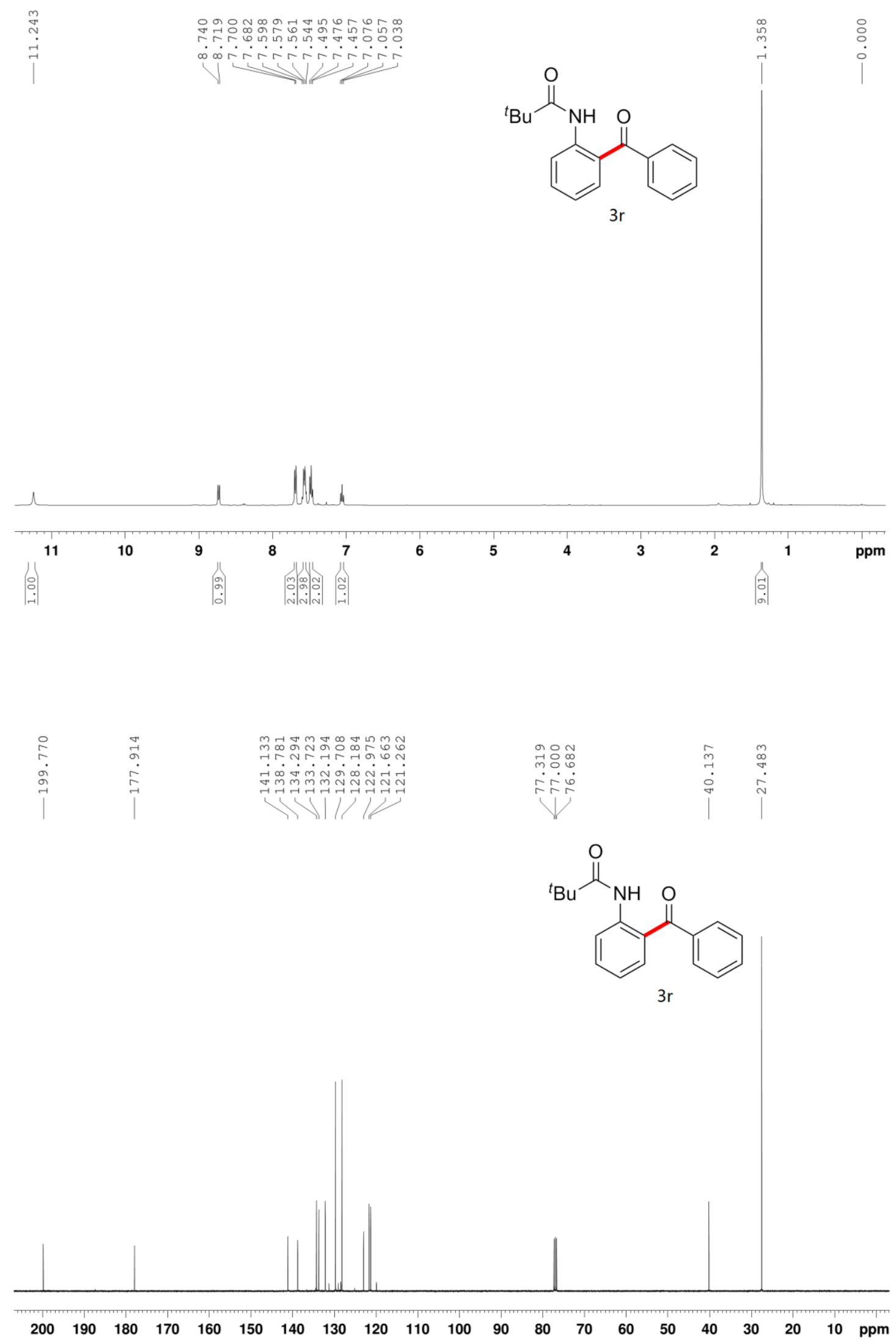


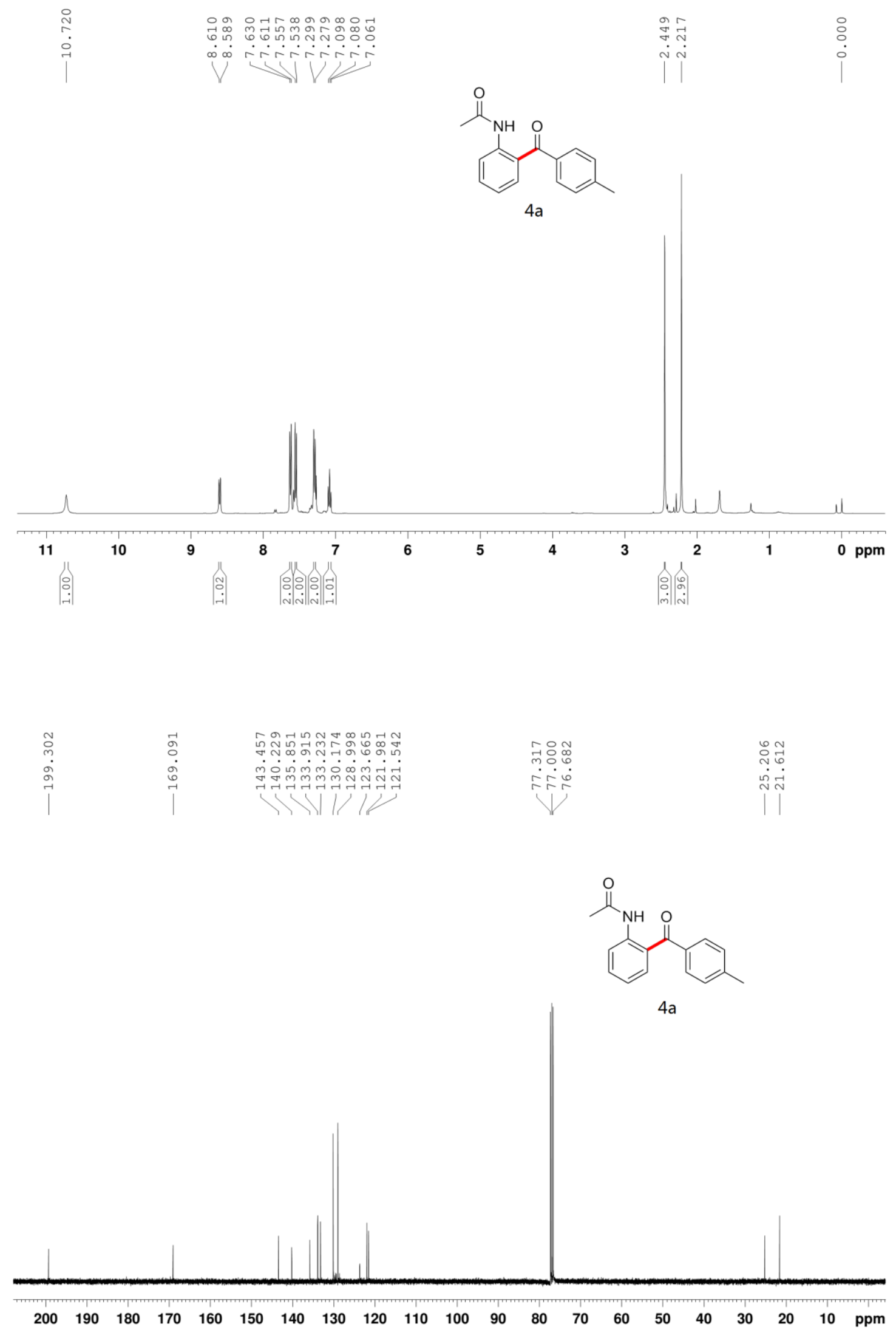



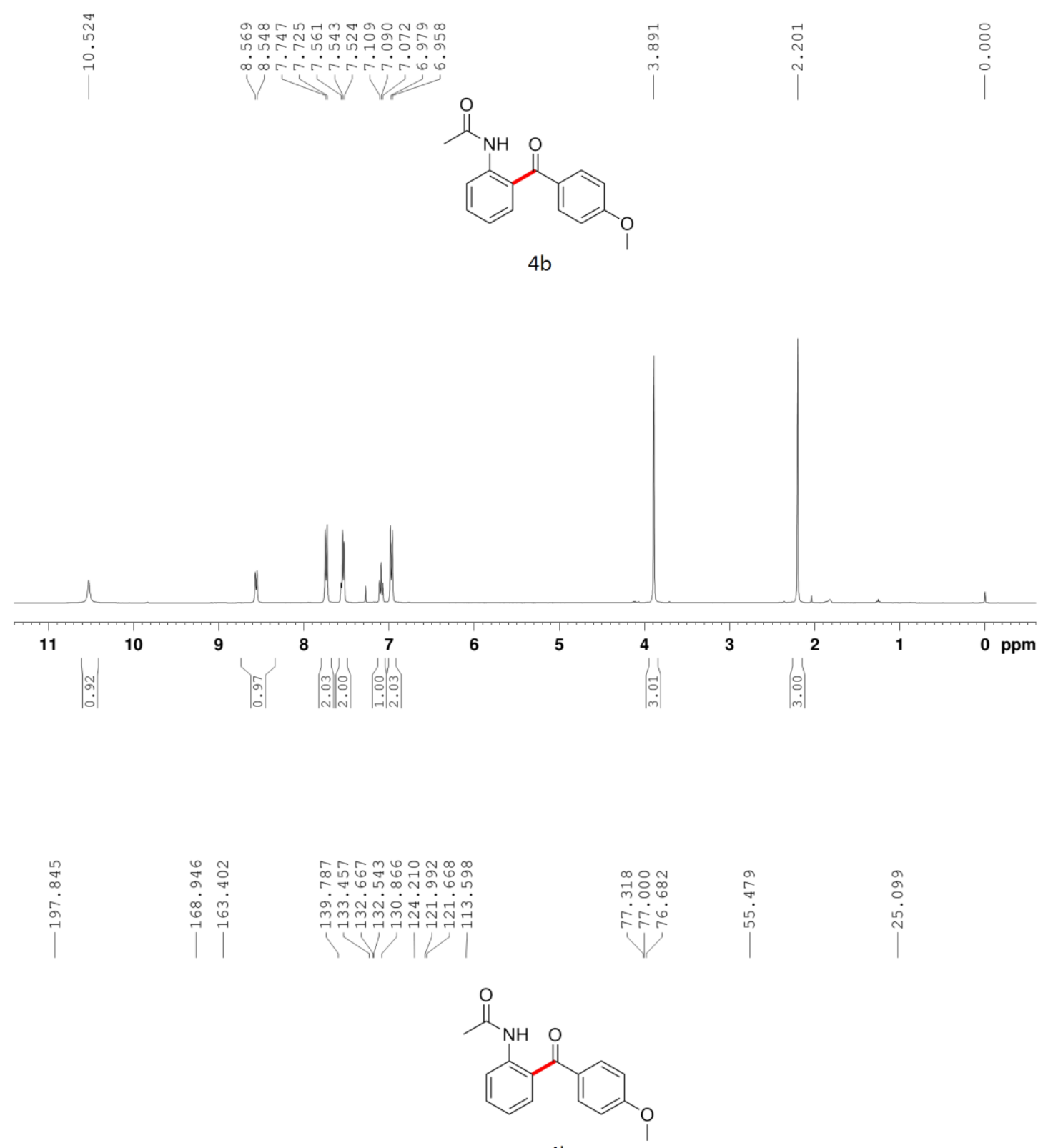

$4 b$

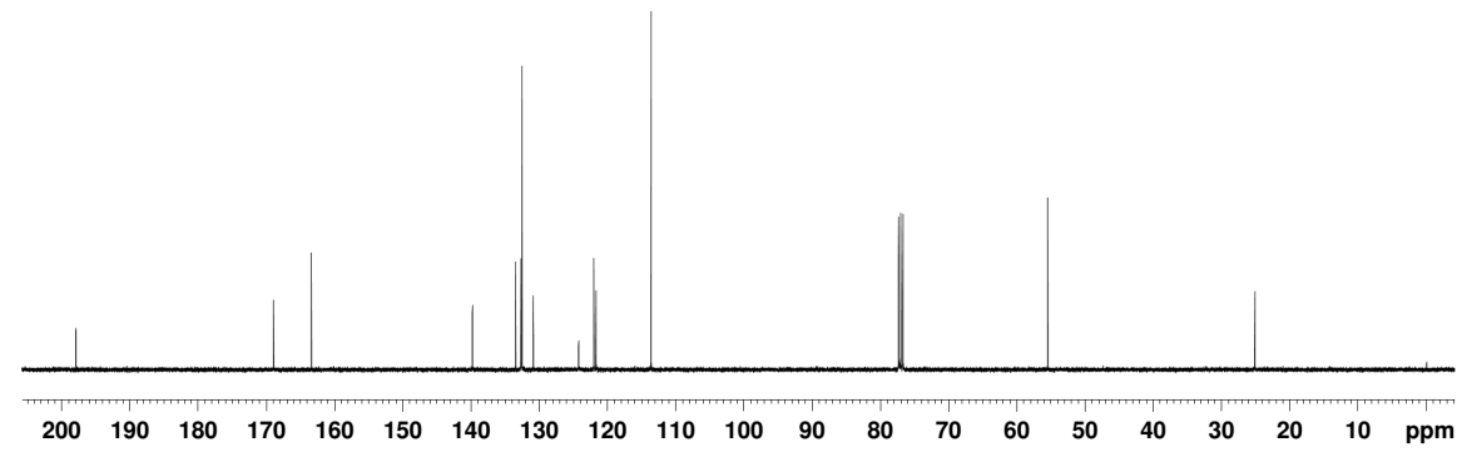



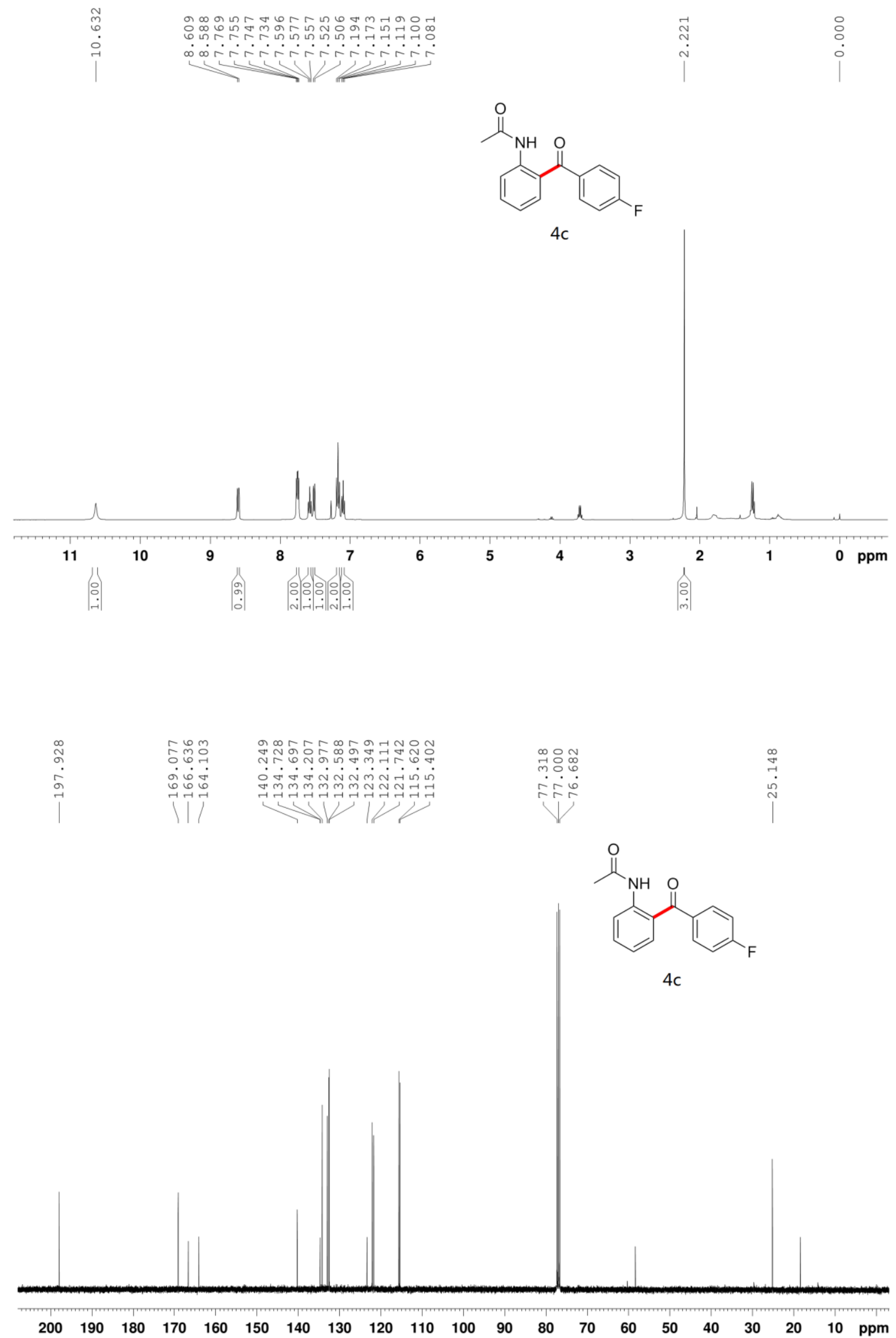


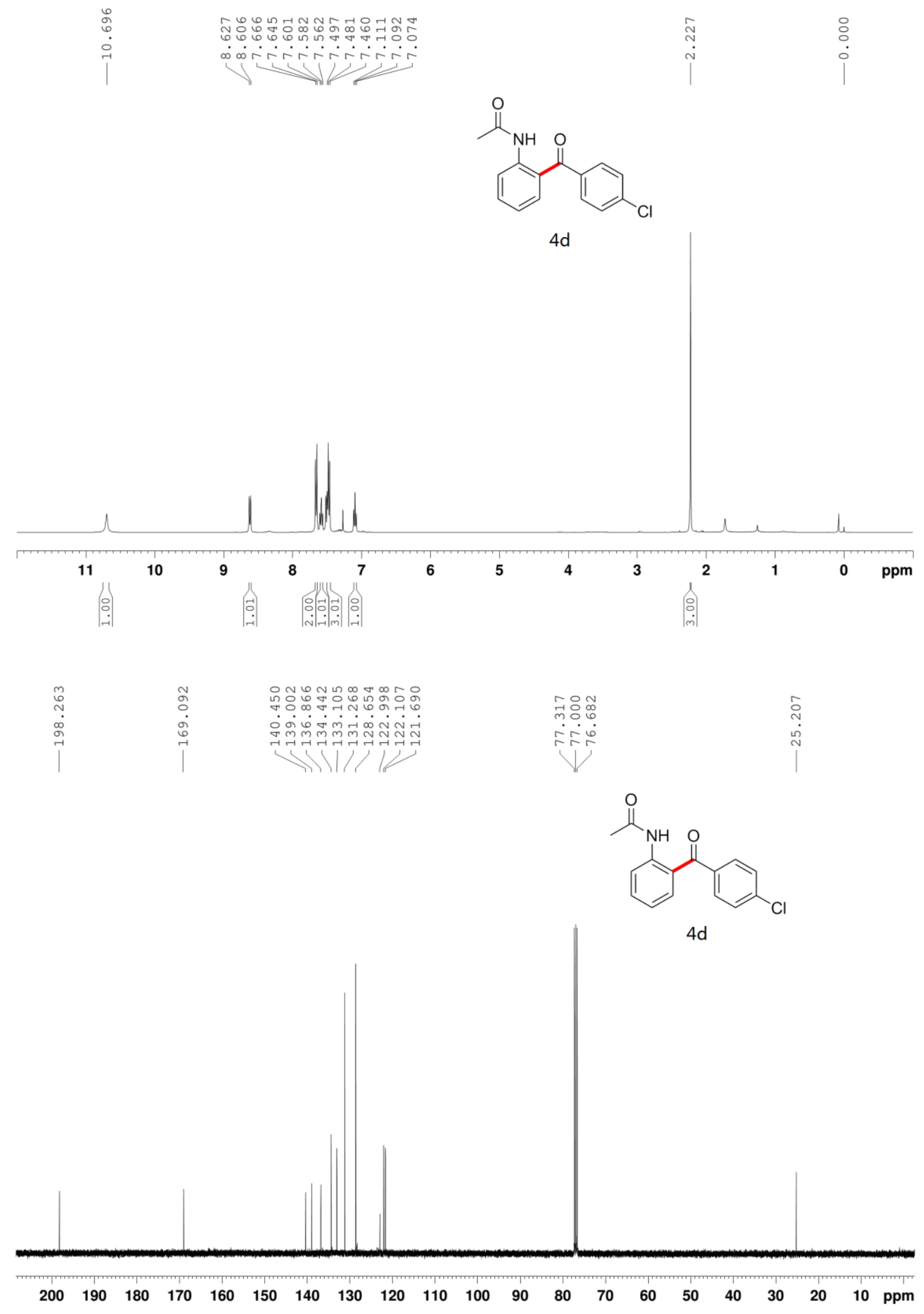




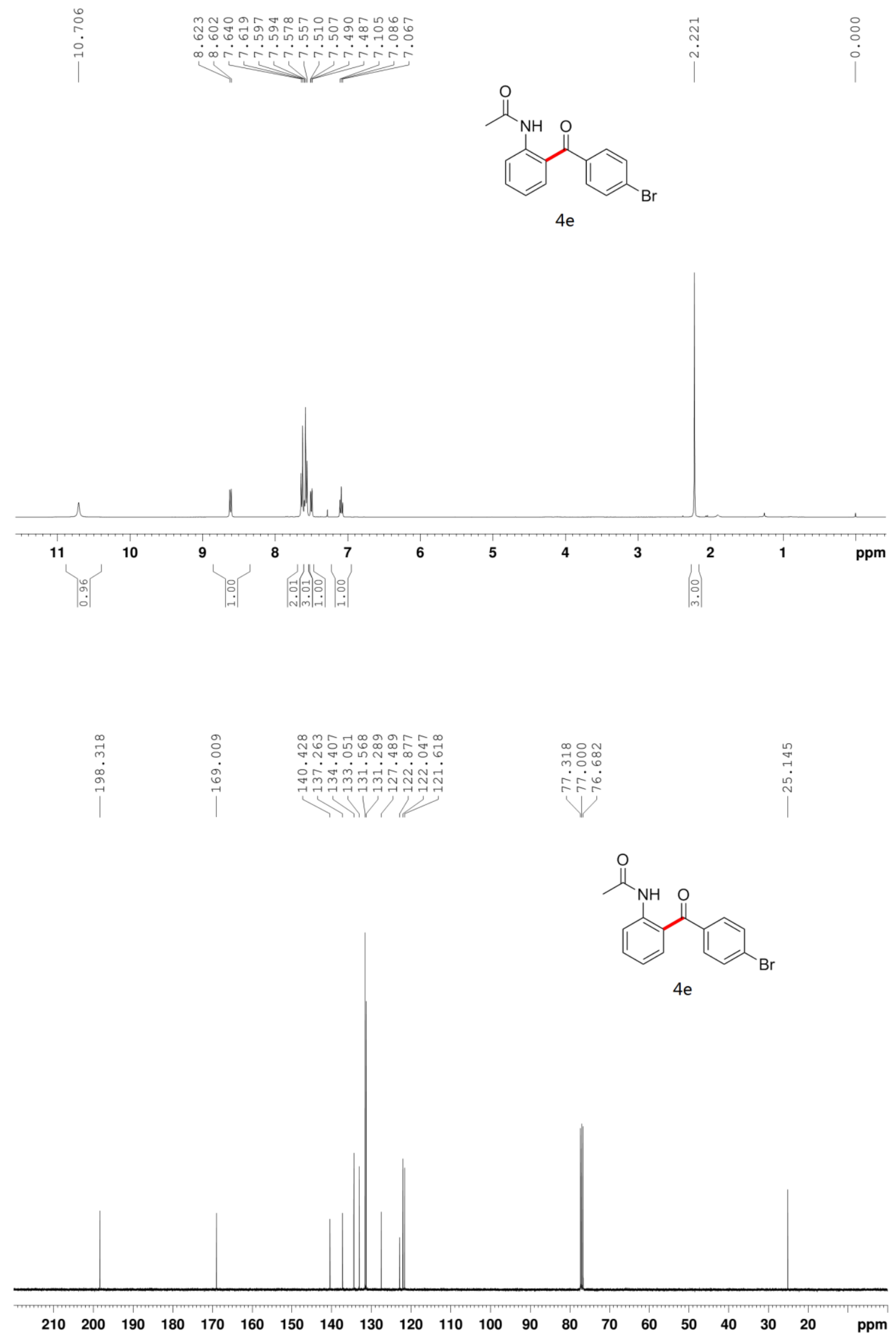




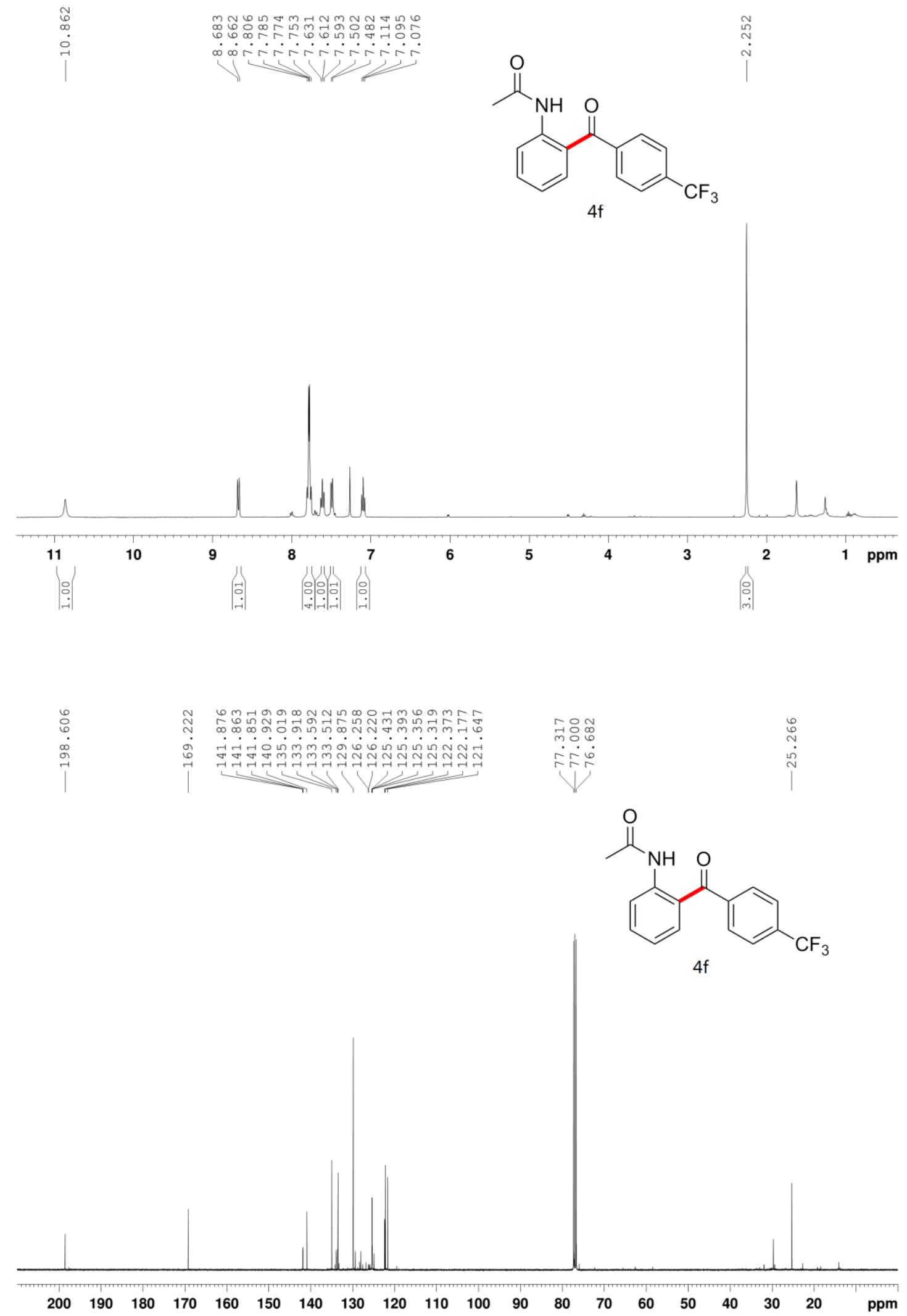




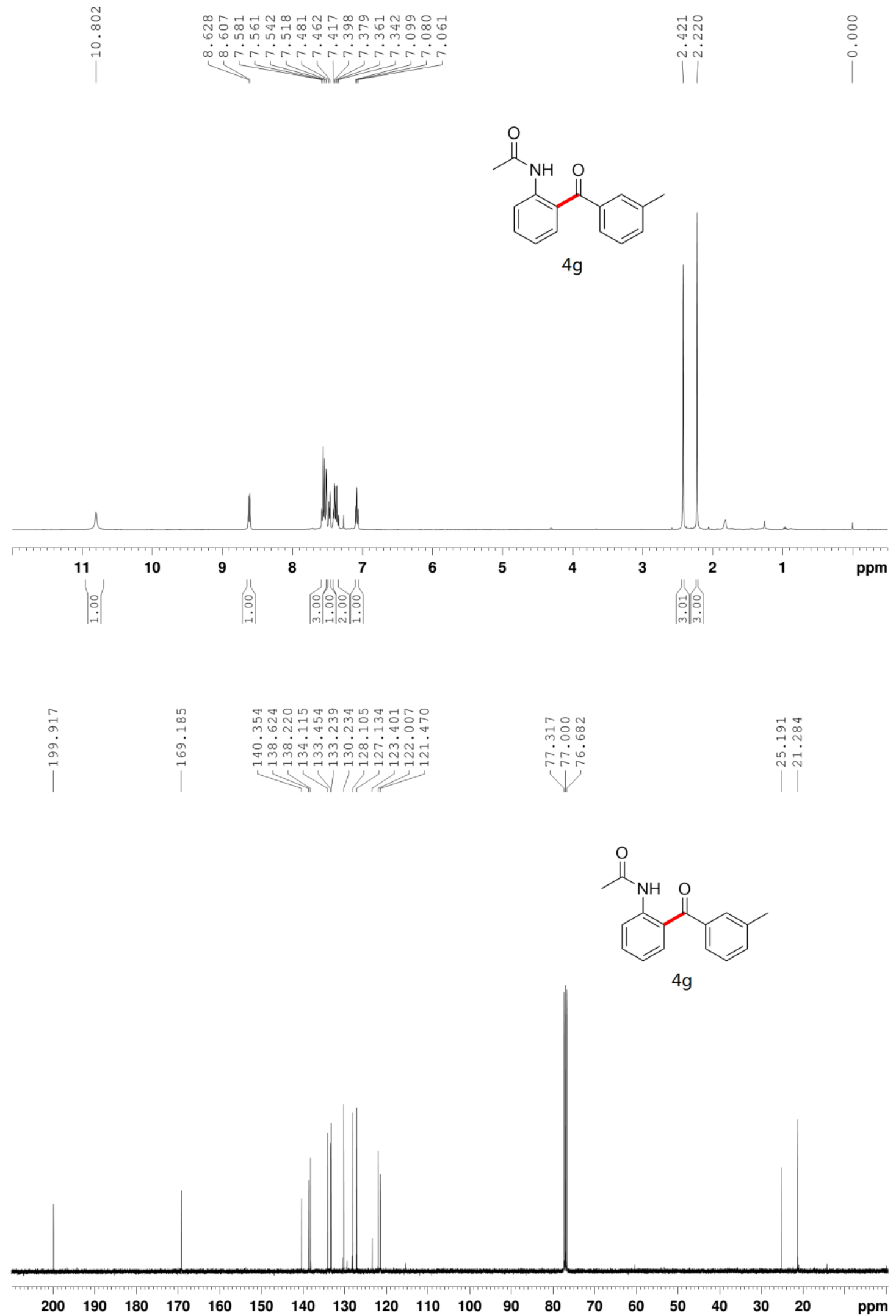



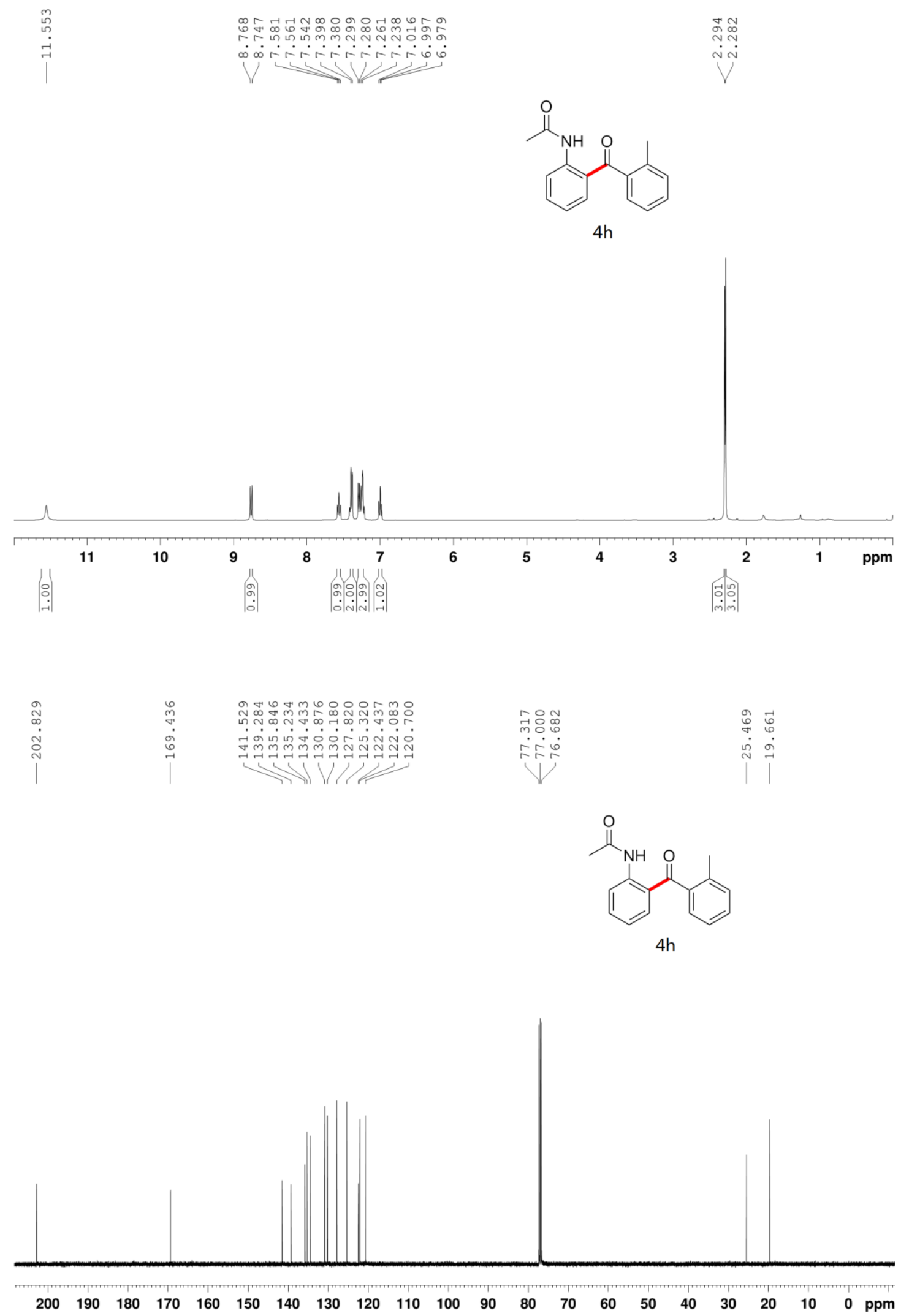


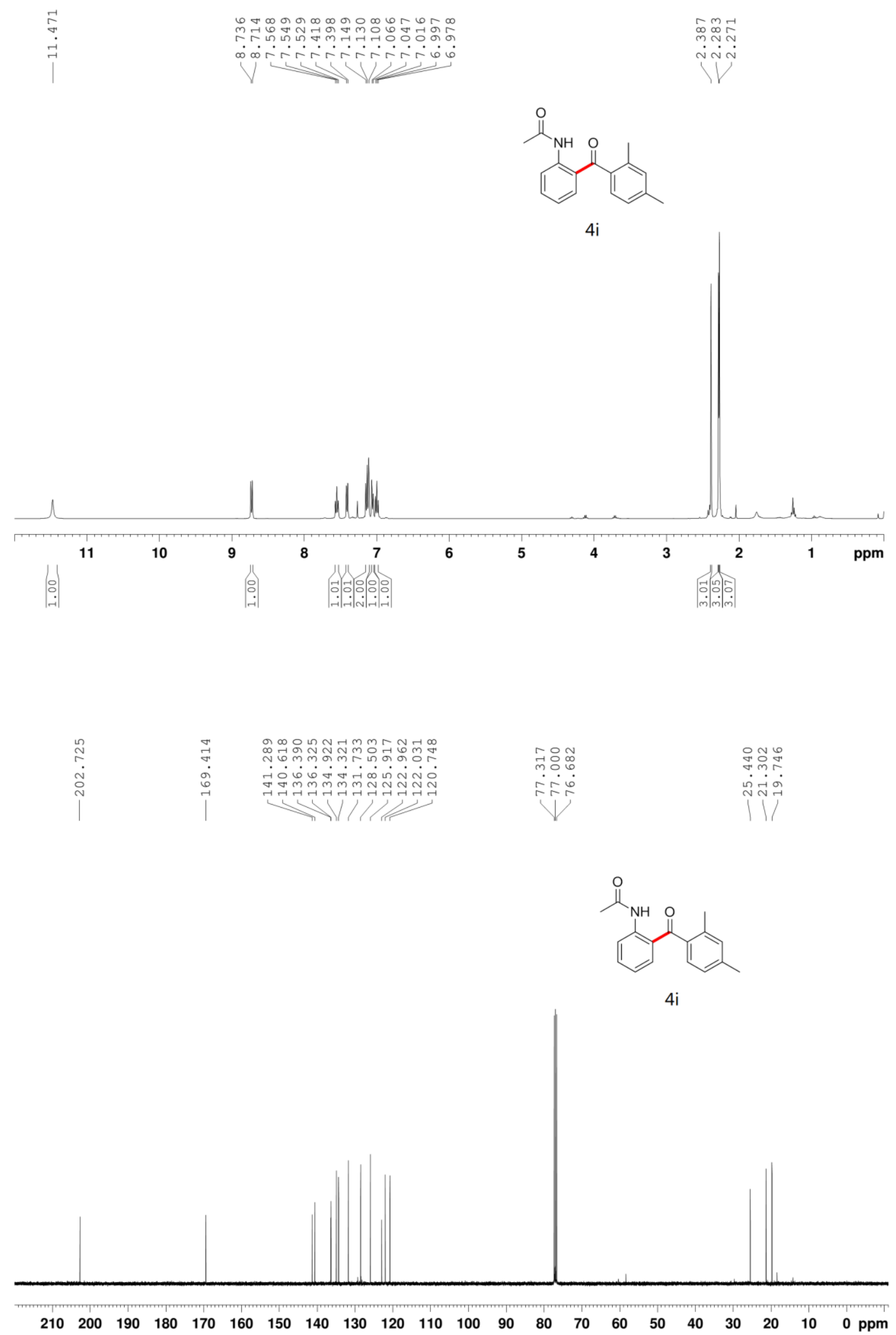



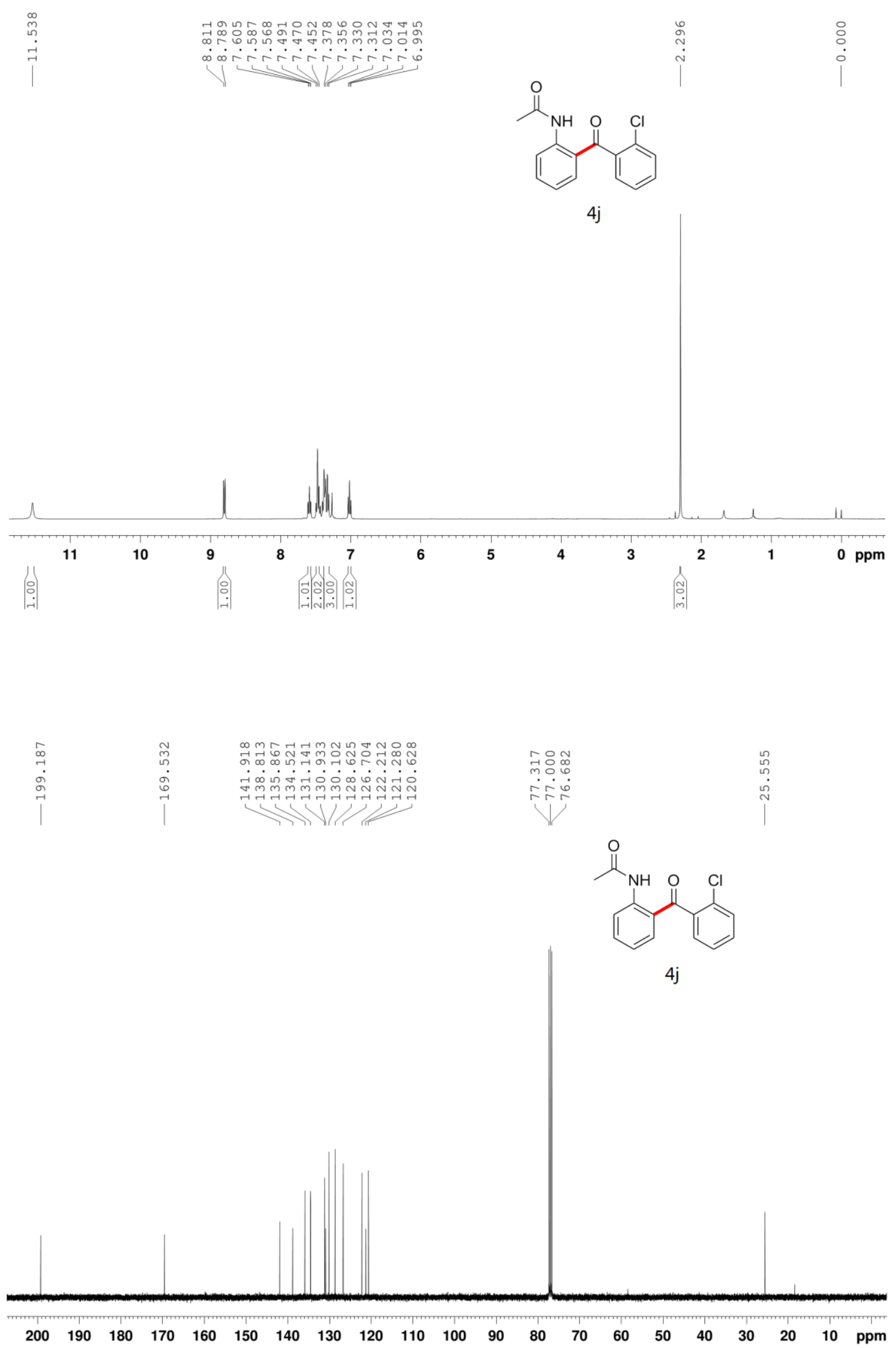

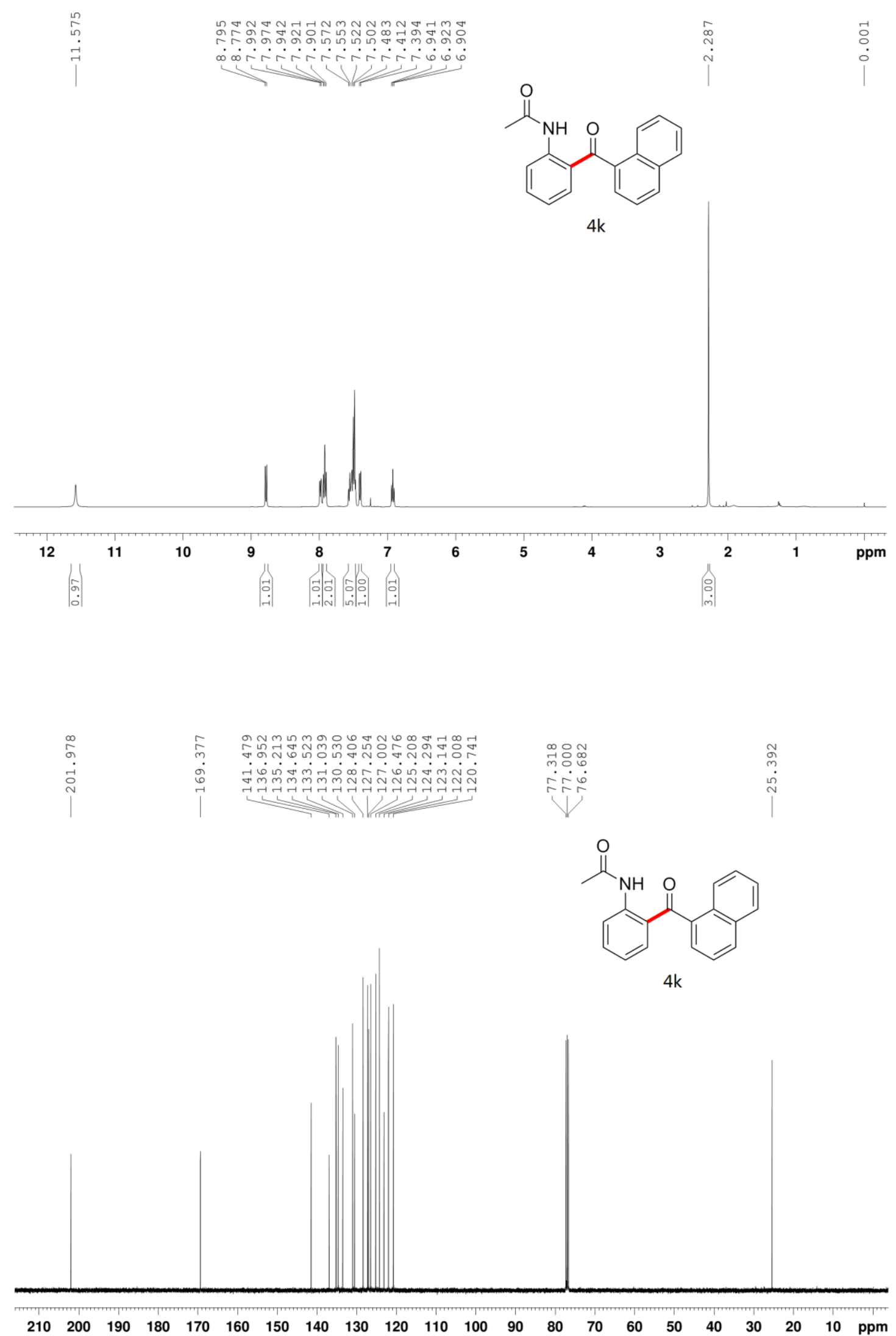

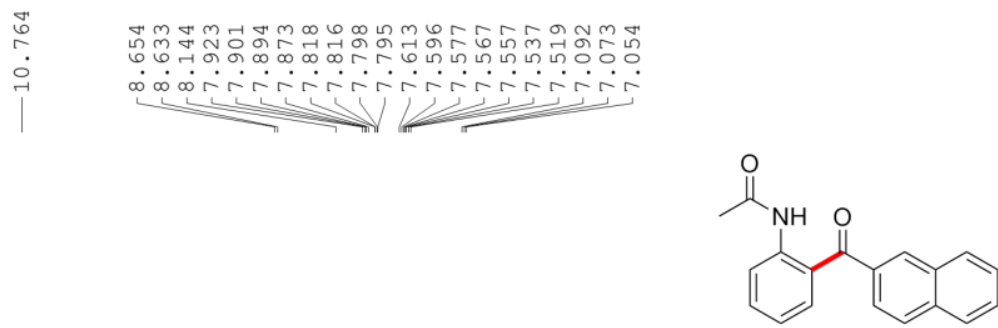

41
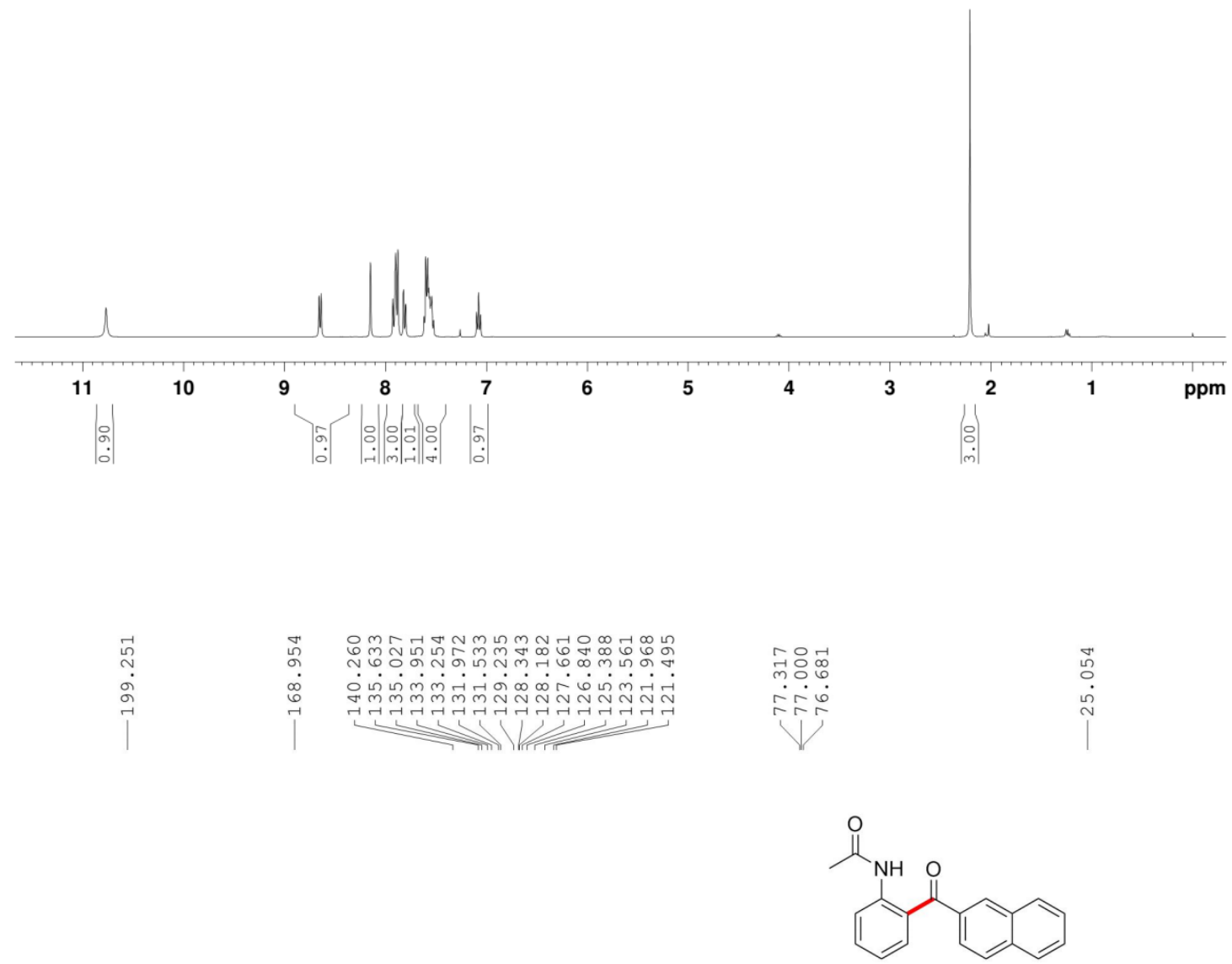

41

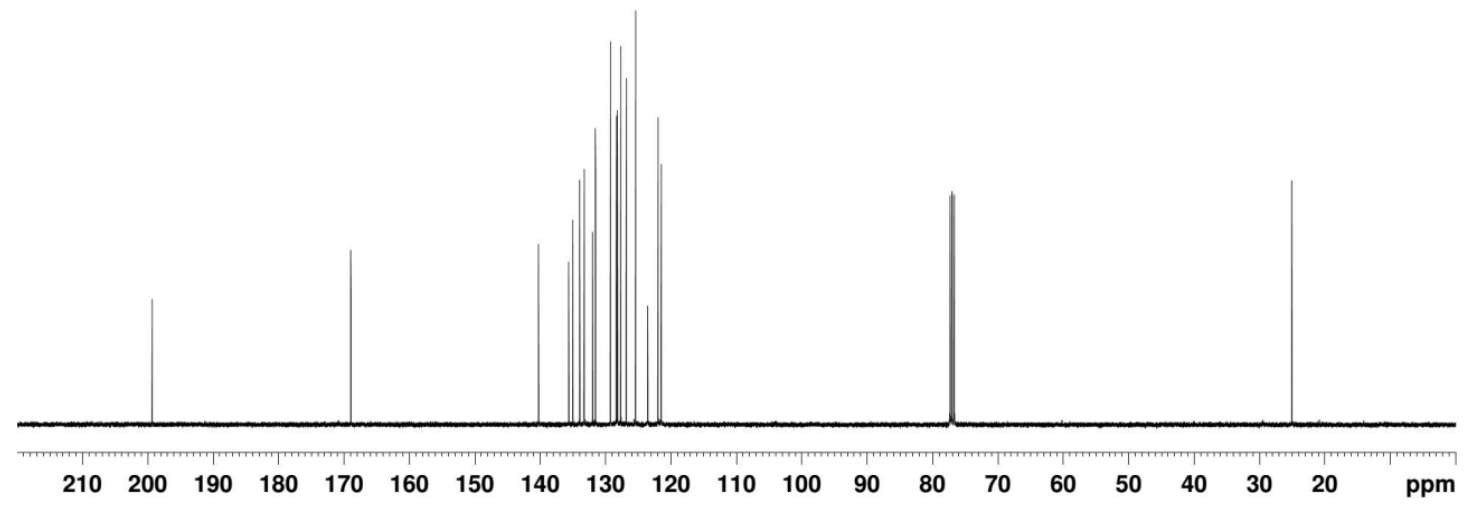




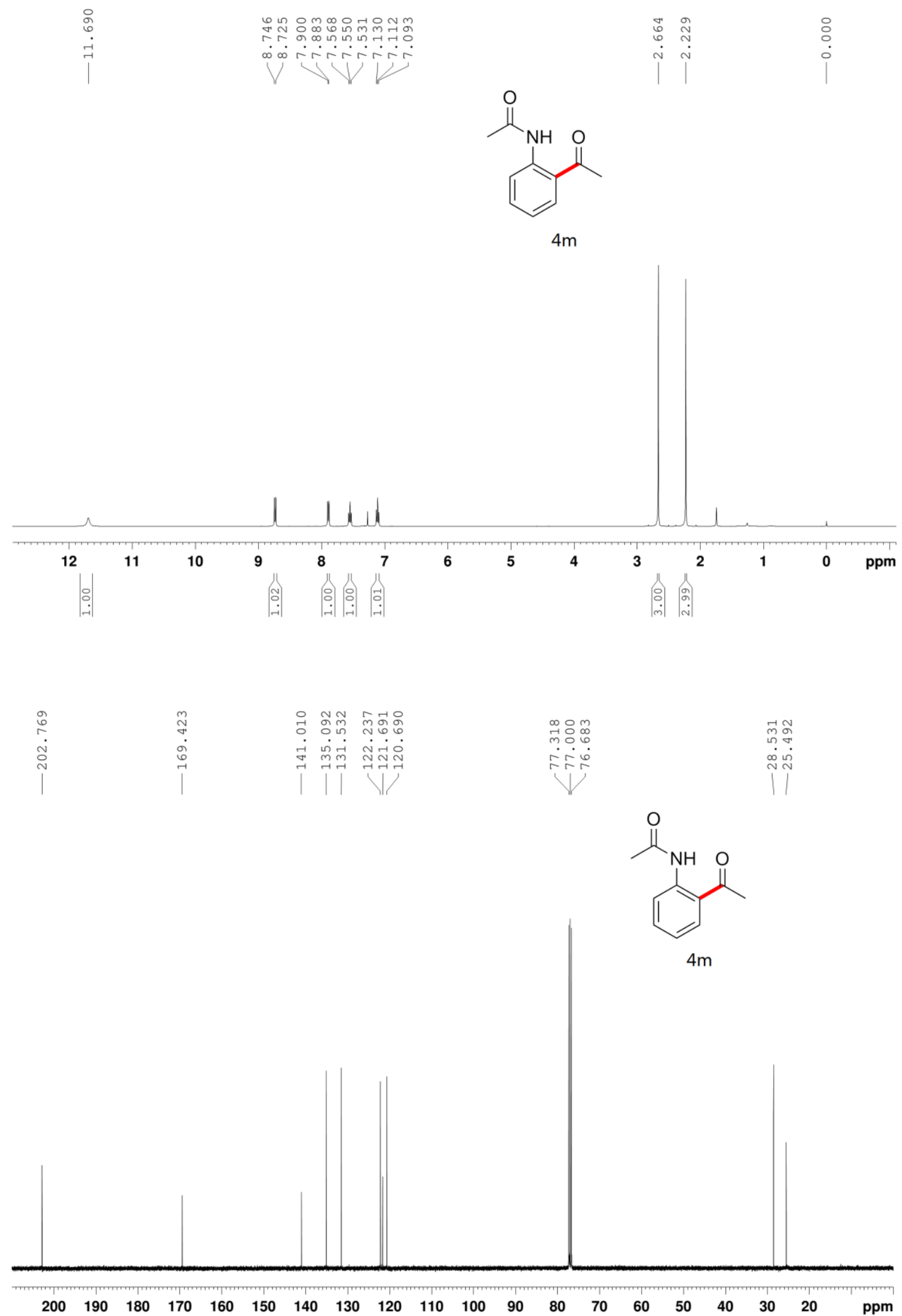




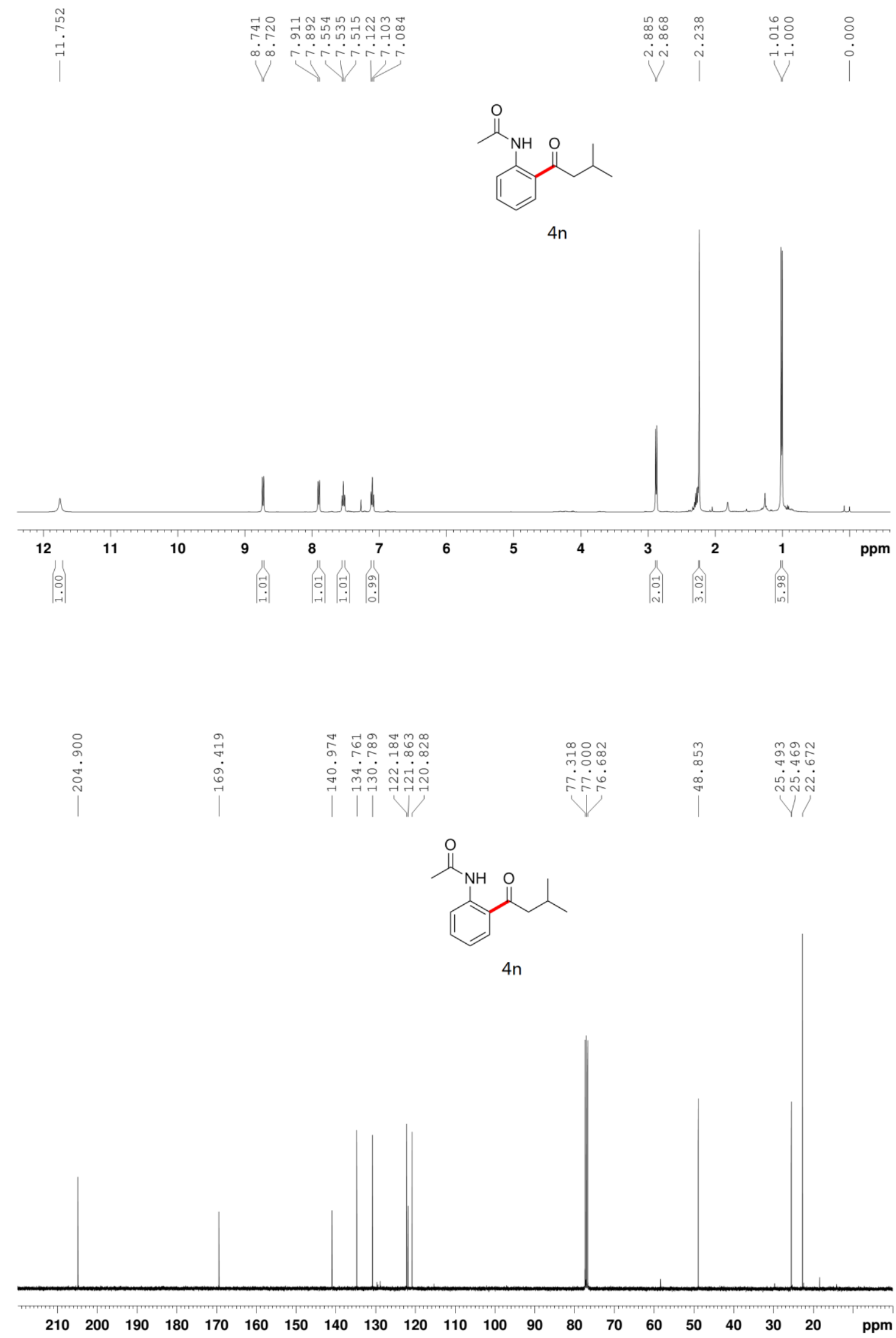




\section{References}

[1] Li, D.-K.; Xu, N.; Zhang, Y.-C.; Wang, L. Chem. Commun. 2014, 50, 14862.

[2] Li, D.-K.; Wang, M.; Liu, J.; Zhao, Q.; Wang, L. Chem. Commun. 2013, 49, 3640.

[3] (a) Zoller, J.; Fabry, D. C.; Ronge, M. A.; Rueping, M. Angew. Chem., Int. Ed. 2014, 53, 13264. (b) Fabry, D. C.; Zoller, J.; RaJa, S.; Rueping, M. Angew. Chem., Int. Ed. 2014, 53, 10228. (c) Fabry, D. C.; Ronge, M. A.; Zoller, J.; Rueping, M. Angew. Chem. Int. Ed. 2015, 54, 2801.

[4] (a) Zhong, J.-J.; Meng, Q.-Y.; Wang, G.-X.; Liu, Q.; Chen, B.; Feng, K.; Tung, C.-H.; Wu, L.-Z. Chem. Eur. J. 2013, 19, 6443. (b) Gao, X.-W.; Meng, Q.-Y.; Xiang, M.; Chen, B.; Feng, K.; Tung, C.-H.; Wu, L.-Z. Adv. Synth. Catal. 2013, $355,2158$.

[5] Tan, H.; Li, H.; Ji, W.; Wang, L. Angew. Chem. Int. Ed. 2015, 54, 8374.

[6] Fang, P.; Li, M.; Ge, H.-B. J. Am. Chem. Soc. 2010, 132, 11898.

[7] Yin, Z.-W.; Sun, P.-P. J. Org. Chem. 2012, 77, 11339.

[8] Szabó, F.; Daru, J.; Simkó, D.; Nagy, T. Z.; Stirling, A.; Novák, Z. Adv. Synth. Catal. 2013, 355, 685 .

[9] Nigel, R.; Helena, T.; Jean, P.; Ludivine, F.; Can, N.; Serge, D.; Christian, M.; Laurent, D. Bioorg. Med. Chem. 2007, 17, 5523.

[10] Yuan, Y.; Chen, D.-T.; Wang, X.-W. Adv. Synth. Catal. 2011, 353, 3373.

[11] Bernd, S.; Nelli, E.; Uwe, S.; Alexandra, K. J. Org. Chem. 2015, 80, 4223. 